\title{
Spectral LFER studies on some sydnone chalcones
}

\section{G. Thirunarayanan ${ }^{1,{ }^{*}}$, G. Vanangamudi ${ }^{2}$, V. Sathiyendiran ${ }^{3}$}

${ }^{1}$ Department of Chemistry, Annamalai University, Annamalainagar-608002, India

${ }^{2} \mathrm{PG}$ and Research Department of Chemistry, Govt. Arts College, C-Mutlur-608102, India

${ }^{3}$ Department of Chemistry, Sourashtra College, Madurai-625004, India.

E-mail address: thirunarayanan.g.10313@annamalaiuniversity.ac.in; drgtnarayanan@gmail.com

Keywords: Sydnone chalcones; IR spectra; NMR spectra; Spectral LFER; Hammett substituent constants

ABSTRACT. A series containing ten (E)-4-(N-(4-cinnamoylphenyl)sulfamoyl)-3-( $p$-tolyl)-1,2,3oxadiazol-3-ium-5-olates were prepared and examined their purities by literature method. The infrared and NMR spectral data were assigned and correlated with Hammett substituent constants and Swain-Lupton's constants using single and multi-regression analysis. From the results of statistical analyses the effects of substituents on the spectral data have been discussed.

\section{INTRODUCTION}

Sydnones are five membered mesoionic dipolar compounds [1]. With reactivity and stability they are fairly different compounds from other aromatics [2]. Sydnones plays on important role in medicinal, heterocyclic and theoretical chemistry [3]. These compounds used as key intermediates for synthesis of organic compounds and possess physiological potentials. They possess many biological activities such as antimicrobial[4], antiviral[5], anti-tumour[6], analgesic[7], anti-inflammatory[8], anthelmintic[9], free-radical scavenging[10, nitric oxide donor[11] and anti-cancer[12]. Sydnone chalcones also possess these properties. Spectroscopic data were useful for prediction of geometry and ground state equilibration of organic compounds[13]. From vibrational spectra the $s$-cis and s-trans conformations of carbonyl compounds were predicted[14]. The chemical shifts of NMR spectra were useful for prediction of geometry of compounds such as $E$ or $Z$ along with coupling constants[15]. Proton chemical shifts splitting patterns used for prediction of spatial arrangement of heterocyclic ring protons in cyclohexane and five membered pyrazolines[16]. Correlation analysis was used for studying reaction speeds, structural conformations of alkenes [17], alkynes [18], $\alpha, \beta$ - unsaturated aldehydes[19], ketones[20], $\omega$-substituted ketones[21] and its esters, halo acyl bromides[22], ${ }^{1} \mathrm{H}$ pyrazoles [23] and its derivatives. Thirunarayanan and Manikandan have studied the dosage of drug analysis using correlation study[ 24]. Thirunarayanan et al, have studied the infrared and NMR spectral correlations of phenazine and quinoxaline derivatives [21, 22]. Mayavel et al.,have studied the spectral correlation of some E-imines [23]. The Qsar and Qpr spectral correlation of some dihyroisoxazoles were reported by Thirunarayanan and Sathiyendiran [25]. Thirunarayanan and his co-workers have studied the effect of substituents on spectral data of some oxazine-2-amines[26]. The spectral correlation of infrared and carbon-13 NMR data of quinoxaline and phenazine derivatives were studied by Thirunaryanan et al. [27, 28]. Senbagam et al., have studied the effects of substituents on some substituted (E)- $N$-benzylidene-4H-1,2,4-triazol-4-amines[29]. Vijayakumar et al., have investigated the spectral correlation analysis on some $(E)$-2-benzylidenehydrazine carbothioamides[30]. The effect of substituents on some hydrazine derivatives were investigated by Rajarajan et al.,[31]. On the complete literature survey, the similar study was not reported with sydnone based chalcones. Hence, the authors have reported first time and taken efforts for the study of spectral correlation of the titled compounds by IR and NMR spectra. 


\section{EXPERIMENTAL}

\subsection{General}

In this present investigations, chemicals used were purchased from Sigma-Aldrich Company Bangalore, India. Infrared spectra $\left(\mathrm{KBr}, 4000-400 \mathrm{~cm}^{-1}\right)$ were recorded on a Briker (Thermo Nicolet) Fourier transform spectrophotometer. The NMR spectra of all pyrazolines were recorded on a Bruker AV400 spectrometer operating at $400 \mathrm{MHz}$ to record $1 \mathrm{H}$ and $100 \mathrm{MHz}$ for $13 \mathrm{C}$ spectra in $\mathrm{CDCl}_{3}$ solvent with TMS as internal standard.

\subsection{Synthesis of $(E)-4-(N-(4-c i n n a m o y l p h e n y l) s u l f a m o y l)-3-(p-t o l y l)-1,2,3-0 x a d i a z o l-3-i u m-5-$ olates}

In this present study, the titled compounds were synthesised and characterized by literature method[32]. The general structure of $(E)-4-(N-(4-$ cinnamoylphenyl)sulfamoyl)-3-( $p$-tolyl)-1,2,3oxadiazol-3-ium-5-olates are shown in Fig.1.

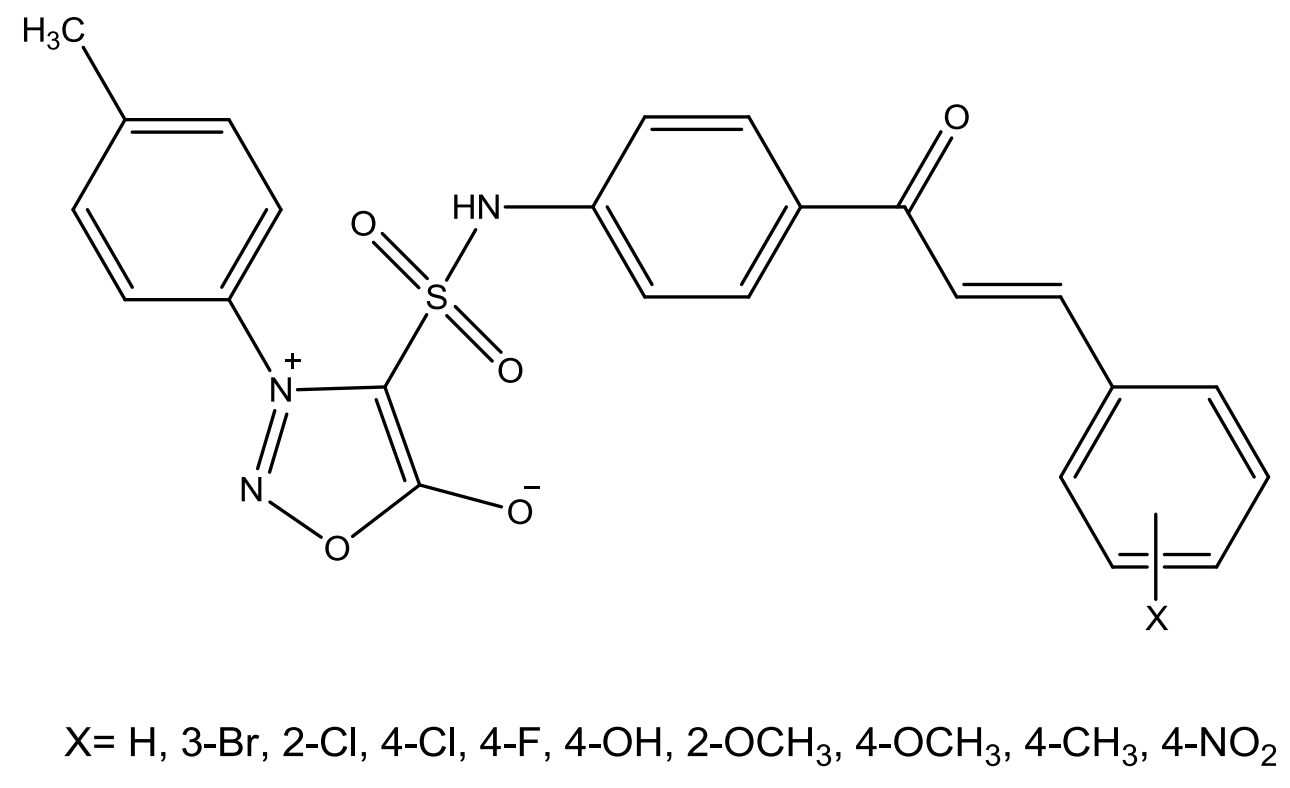

Fig. 1. The general structure of (E)-4-( $N$-(4-cinnamoylphenyl)sulfamoyl)-3-(p-tolyl)-1,2,3oxadiazol-3-ium-5-olates

\section{RESULTS AND DISCUSSION}

\subsection{Correlation analysis with IR spectral data}

Infrared spectra of synthesized $(E)-4-(N$-(4-cinnamoylphenyl)sulfamoyl)-3-( $p$-tolyl)-1,2,3oxadiazol-3-ium-5-olates, the characteristic stretches $\left(v, \mathrm{~cm}^{-1}\right)$ of $\mathrm{CO}_{s-c i s, ~ s-t r a n s}, \mathrm{CH}_{i p},{ }_{o p}, \mathrm{CH}=\mathrm{CH}_{o p}$, $\mathrm{C}=\mathrm{C}_{o p}, \mathrm{NH}, \mathrm{SO}_{2}$, and $\mathrm{CO}_{\text {syd }}$ have been assigned and tabulated in Table 1. Using Hammett equation and single and multi-regression analysis[13-16, 20-31] of these data with Hammett substituent constants and Swain-Lupton's [33] parameters. In this correlation, the Hammett equation was taken in the form as(1). 
Table 1. The infrared characteristics vibrations $\left(v, \mathrm{~cm}^{-1}\right)$ of $(E)-4-(N-(4-$ cinnamoylphenyl $)$ sulfamoyl)-3-( $p$-tolyl)-1,2,3-oxadiazol-3-ium-5-olates

\begin{tabular}{|l|l|l|l|l|l|l|l|l|l|l|}
\hline Entry & $\mathrm{X}$ & $\mathrm{CO}_{s-c i s}$ & $\mathrm{CO}_{s-\text { trans }}$ & $\mathrm{CH}_{i p}$ & $\mathrm{CH}_{o p}$ & $\mathrm{CH}=\mathrm{CH}_{o p}$ & $\mathrm{C}=\mathrm{C}_{o p}$ & $\mathrm{NH}$ & $\mathrm{SO}_{2}$ & $\mathrm{CO}_{s v d}$ \\
\hline 1 & $\mathrm{H}$ & 1618 & 1610 & 1175 & 763 & 1048 & 576 & 3315 & 1327 & 1756 \\
\hline 2 & $3-\mathrm{Br}$ & 1628 & 1595 & 1176 & 767 & 1046 & 570 & 3322 & 1318 & 1758 \\
\hline 3 & $2-\mathrm{Cl}$ & 1620 & 1594 & 1177 & 767 & 1046 & 570 & 3335 & 1320 & 1750 \\
\hline 4 & $4-\mathrm{Cl}$ & 1621 & 1601 & 1174 & 765 & 1044 & 571 & 3536 & 1319 & 1754 \\
\hline 5 & $4-\mathrm{F}$ & 1602 & 1631 & 1174 & 765 & 1045 & 572 & 3311 & 1322 & 1753 \\
\hline 6 & $4-\mathrm{OH}$ & 1623 & 1594 & 1174 & 764 & 1044 & 572 & 3308 & 1310 & 1755 \\
\hline 7 & $2-\mathrm{OCH}_{3}$ & 1621 & 1593 & 1175 & 763 & 1044 & 572 & 3312 & 1324 & 1754 \\
\hline 8 & $4-\mathrm{OCH}_{3}$ & 1622 & 1595 & 1170 & 757 & 1043 & 569 & 3329 & 1316 & 1748 \\
\hline 9 & $4-\mathrm{CH}_{3}$ & 1648 & 1606 & 1168 & 758 & 1042 & 569 & 3308 & 1318 & 1752 \\
\hline 10 & $4-\mathrm{NO}_{2}$ & 1654 & 1608 & 1172 & 768 & 1053 & 578 & 3347 & 1328 & 1786 \\
\hline
\end{tabular}

$v=v_{0}+\rho \sigma$

where $v_{0}$ is the unsubstitued system.

the obtained statistical results are tabulated in Table 2. From the Table 2, the $\mathrm{CO}_{s-c i s}$ stretches $(v$, $\mathrm{cm}^{-1}$ ) gave satisfactory correlations with Hammett $\sigma_{\mathrm{I}}, \sigma_{\mathrm{R}}$, constants, F and R parameters positive $\rho$ values excluding $4-\mathrm{CH}_{3}$ and $4-\mathrm{NO}_{2}$ substituents. This $\rho$ value indicates that, the normal substituent effects operate in all systems. The Hammett $\sigma$ and $\sigma^{+}$constants produced poor correlation. The $\mathrm{CO}_{\text {s-trans }}$ stretches $\left(\mathrm{v}, \mathrm{cm}^{-1}\right)$ of the sydnone chalcones produced satisfactory correlation with Hammett $\sigma, \sigma^{+}, \sigma_{\mathrm{R}}$ constants and $\mathrm{R}$ parameters excluding $\mathrm{H}, 2-\mathrm{Cl}$, and $2-\mathrm{OCH}_{3}$ substituents. The inductive and field components of the substituents produced poor correlations. The failure in correlation was the inability of prediction of effects of substituents on the stretches and attributed with the resonance-conjugative structure as illustrated in Fig. 2.

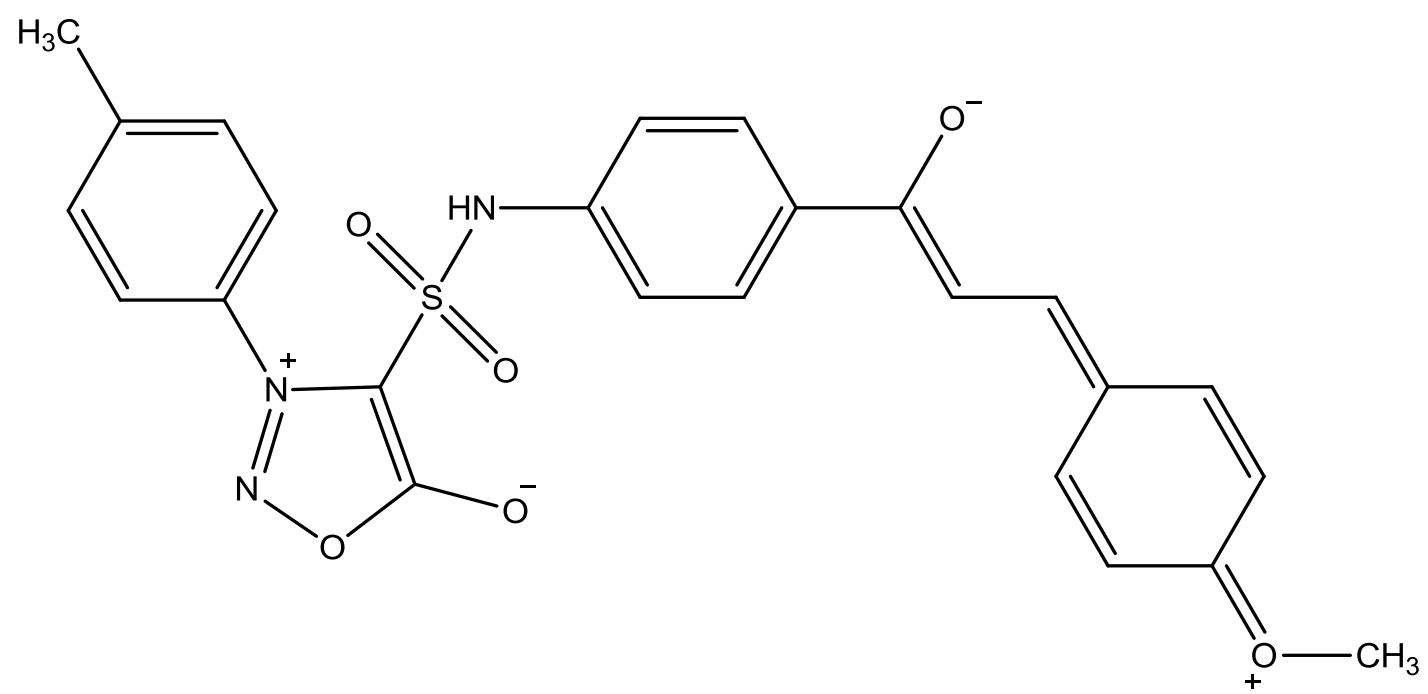

Fig. 2. The resonance-conjugative structure 
Table 2. Results of statistical analysis of infrared vibrations $\left(v, \mathrm{~cm}^{-1}\right)$ of (E)-4-(N-(4cinnamoylphenyl)sulfamoyl)-3-( $p$-tolyl)-1,2,3-oxadiazol-3-ium-5-olates with Hammett substituent constants, $\mathrm{F}$ and $\mathrm{R}$ parameters.

\begin{tabular}{|c|c|c|c|c|c|c|c|}
\hline Frequency & Constant & $\mathrm{r}$ & I & $\rho$ & $\mathrm{s}$ & $\mathrm{n}$ & Correlated derivatives \\
\hline \multirow[t]{6}{*}{$\mathrm{CO}_{\text {s-cis }}$} & $\sigma$ & 0.728 & 1629.35 & 13.061 & 20.05 & 10 & $\mathrm{H}, 3-\mathrm{Br}, 2-\mathrm{Cl}, 4-\mathrm{Cl}, 4-\mathrm{F}, 4-\mathrm{OH}, 2-\mathrm{OCH}_{3}, 4-\mathrm{OCH}_{3}, 4-\mathrm{CH}_{3}, 4-\mathrm{NO}_{2}$ \\
\hline & $\sigma^{+}$ & 0.736 & 1652.35 & 9.572 & 19.68 & 10 & $\mathrm{H}, 3-\mathrm{Br}, 2-\mathrm{Cl}, 4-\mathrm{Cl}, 4-\mathrm{F}, 4-\mathrm{OH}, 2-\mathrm{OCH}_{3}, 4-\mathrm{OCH}_{3}, 4-\mathrm{CH}_{3}, 4-\mathrm{NO}_{2}$ \\
\hline & $\sigma_{1}$ & 0.905 & 1630.86 & 43.562 & 17.36 & 9 & $\mathrm{H}, 3-\mathrm{Br}, 2-\mathrm{Cl}, 4-\mathrm{Cl}, 4-\mathrm{F}, 4-\mathrm{OH}, 2-\mathrm{OCH}_{3}, 4-\mathrm{OCH}_{3}, 4-\mathrm{CH}_{3}$ \\
\hline & $\sigma_{R}$ & 0.900 & 1644.88 & 65.836 & 12.26 & 10 & $\mathrm{H}, 3-\mathrm{Br}, 2-\mathrm{Cl}, 4-\mathrm{Cl}, 4-\mathrm{F}, 4-\mathrm{OH}, 2-\mathrm{OCH}_{3}, 4-\mathrm{OCH}_{3}, 4-\mathrm{CH}_{3}, 4-\mathrm{NO}_{2}$ \\
\hline & $\mathrm{F}$ & 0.905 & 1646.36 & 47.262 & 12.76 & 9 & $\mathrm{H}, 3-\mathrm{Br}, 2-\mathrm{Cl}, 4-\mathrm{Cl}, 4-\mathrm{F}, 4-\mathrm{OH}, 2-\mathrm{OCH}_{3}, 4-\mathrm{OCH}_{3}, 4-\mathrm{CH}_{3}$, \\
\hline & $\mathrm{R}$ & 0.900 & 1642.35 & 35.682 & 16.84 & 10 & $\mathrm{H}, 3-\mathrm{Br}, 2-\mathrm{Cl}, 4-\mathrm{Cl}, 4-\mathrm{F}, 4-\mathrm{OH}, 2-\mathrm{OCH}_{3}, 4-\mathrm{OCH}_{3}, 4-\mathrm{CH}_{3}, 4-\mathrm{NO}_{2}$ \\
\hline \multirow[t]{6}{*}{$\mathrm{CO}_{\text {s-trans }}$} & $\sigma$ & 0.906 & 1599.13 & 14.539 & 6.55 & 9 & $\mathrm{H}, 3-\mathrm{Br}, 4-\mathrm{Cl}, 4-\mathrm{F}, 4-\mathrm{OH}, 2-\mathrm{OCH}_{3}, 4-\mathrm{OCH}_{3}, 4-\mathrm{CH}_{3}, 4-\mathrm{NO}_{2}$ \\
\hline & $\sigma^{+}$ & 0.905 & 1600.00 & 8.439 & 7.16 & 10 & $\mathrm{H}, 3-\mathrm{Br}, 2-\mathrm{Cl}, 4-\mathrm{Cl}, 4-\mathrm{F}, 4-\mathrm{OH}, 2-\mathrm{OCH}_{3}, 4-\mathrm{OCH}_{3}, 4-\mathrm{CH}_{3}, 4-\mathrm{NO}_{2}$ \\
\hline & $\sigma_{1}$ & 0.804 & 1599.20 & 1.578 & 8.69 & 10 & $\mathrm{H}, 3-\mathrm{Br}, 2-\mathrm{Cl}, 4-\mathrm{Cl}, 4-\mathrm{F}, 4-\mathrm{OH}, 2-\mathrm{OCH}_{3}, 4-\mathrm{OCH}_{3}, 4-\mathrm{CH}_{3}, 4-\mathrm{NO}_{2}$ \\
\hline & $\sigma_{\mathrm{R}}$ & 0.909 & 1607.42 & 30.856 & 4.15 & 10 & $\mathrm{H}, 3-\mathrm{Br}, 2-\mathrm{Cl}, 4-\mathrm{Cl}, 4-\mathrm{F}, 4-\mathrm{OH}, 2-\mathrm{OCH}_{3}, 4-\mathrm{OCH}_{3}, 4-\mathrm{CH}_{3}, 4-\mathrm{NO}_{2}$ \\
\hline & $\mathrm{F}$ & 0.800 & 1599.92 & 0.355 & 8.71 & 10 & $\mathrm{H}, 3-\mathrm{Br}, 2-\mathrm{Cl}, 4-\mathrm{Cl}, 4-\mathrm{F}, 4-\mathrm{OH}, 2-\mathrm{OCH}_{3}, 4-\mathrm{OCH}_{3}, 4-\mathrm{CH}_{3}, 4-\mathrm{NO}_{2}$ \\
\hline & $\mathrm{R}$ & 0.908 & 1607.25 & 24.854 & 4.68 & 10 & $\mathrm{H}, 3-\mathrm{Br}, 2-\mathrm{Cl}, 4-\mathrm{Cl}, 4-\mathrm{F}, 4-\mathrm{OH}, 2-\mathrm{OCH}_{3}, 4-\mathrm{OCH}_{3}, 4-\mathrm{CH}_{3}, 4-\mathrm{NO}_{2}$ \\
\hline \multirow[t]{6}{*}{$\mathrm{CH}_{i p}$} & $\sigma$ & 0.906 & 1173.82 & 10.280 & 4.73 & 8 & $\mathrm{H}, 3-\mathrm{Br}, 2-\mathrm{Cl}, 4-\mathrm{Cl}, 4-\mathrm{F}, 4-\mathrm{OH}, 4-\mathrm{OCH}_{3}, 4-\mathrm{NO}_{2}$ \\
\hline & $\sigma^{+}$ & 0.906 & 1174.82 & 6.599 & 4.89 & 8 & $\mathrm{H}, 3-\mathrm{Br}, 2-\mathrm{Cl}, 4-\mathrm{Cl}, 4-\mathrm{F}, 4-\mathrm{OH}, 4-\mathrm{OCH}_{3}, 4-\mathrm{NO}_{2}$ \\
\hline & $\sigma_{1}$ & 0.917 & 1167.49 & 20.086 & 3.97 & 9 & 3- $\mathrm{Br}, 2-\mathrm{Cl}, 4-\mathrm{Cl}, 4-\mathrm{F}, 4-\mathrm{OH}, 2-\mathrm{OCH}_{3}, 4-\mathrm{OCH}_{3}, 4-\mathrm{CH}_{3}, 4-\mathrm{NO}_{2}$ \\
\hline & $\sigma_{R}$ & 0.821 & 1175.57 & 5.140 & 6.09 & 10 & $\mathrm{H}, 3-\mathrm{Br}, 2-\mathrm{Cl}, 4-\mathrm{Cl}, 4-\mathrm{F}, 4-\mathrm{OH}, 2-\mathrm{OCH}_{3}, 4-\mathrm{OCH}_{3}, 4-\mathrm{CH}_{3}, 4-\mathrm{NO}_{2}$ \\
\hline & $\mathrm{F}$ & 0.902 & 1169.92 & 17.701 & 4.34 & 9 & $3-\mathrm{Br}, 2-\mathrm{Cl}, 4-\mathrm{Cl}, 4-\mathrm{F}, 4-\mathrm{OH}, 2-\mathrm{OCH}_{3}, 4-\mathrm{OCH}_{3}, 4-\mathrm{CH}_{3}, 4-\mathrm{NO}_{2}$ \\
\hline & $\mathrm{R}$ & 0.824 & 1175.93 & 5.257 & 6.22 & 10 & $\mathrm{H}, 3-\mathrm{Br}, 2-\mathrm{Cl}, 4-\mathrm{Cl}, 4-\mathrm{F}, 4-\mathrm{OH}, 2-\mathrm{OCH}_{3}, 4-\mathrm{OCH}_{3}, 4-\mathrm{CH}_{3}, 4-\mathrm{NO}_{2}$ \\
\hline \multirow[t]{6}{*}{$\mathrm{CH}_{o p}$} & $\sigma$ & 0.926 & 764.13 & 7.854 & 3.45 & 9 & $\mathrm{H}, 3-\mathrm{Br}, 2-\mathrm{Cl}, 4-\mathrm{Cl}, 4-\mathrm{F}, 2-\mathrm{OCH}_{3}, 4-\mathrm{OCH}_{3}, 4-\mathrm{CH}_{3}, 4-\mathrm{NO}_{2}$ \\
\hline & $\sigma^{+}$ & 0.907 & 765.18 & 4.812 & 3.68 & 9 & $\mathrm{H}, 3-\mathrm{Br}, 2-\mathrm{Cl}, 4-\mathrm{Cl}, 4-\mathrm{F}, 2-\mathrm{OCH}_{3}, 4-\mathrm{OCH}_{3}, 4-\mathrm{CH}_{3}, 4-\mathrm{NO}_{2}$ \\
\hline & $\sigma_{1}$ & 0.907 & 760.05 & 13.991 & 3.21 & 10 & $\mathrm{H}, 3-\mathrm{Br}, 2-\mathrm{Cl}, 4-\mathrm{Cl}, 4-\mathrm{F}, 4-\mathrm{OH}, 2-\mathrm{OCH}_{3}, 4-\mathrm{OCH}_{3}, 4-\mathrm{CH}_{3}, 4-\mathrm{NO}_{2}$ \\
\hline & $\sigma_{R}$ & 0.831 & 766.25 & 5.884 & 4.39 & 10 & $\mathrm{H}, 3-\mathrm{Br}, 2-\mathrm{Cl}, 4-\mathrm{Cl}, 4-\mathrm{F}, 4-\mathrm{OH}, 2-\mathrm{OCH}_{3}, 4-\mathrm{OCH}_{3}, 4-\mathrm{CH}_{3}, 4-\mathrm{NO}_{2}$ \\
\hline & F & 0.906 & 760.74 & 11.272 & 3.66 & 9 & $\mathrm{H}, 3-\mathrm{Br}, 2-\mathrm{Cl}, 4-\mathrm{Cl}, 4-\mathrm{OH}, 2-\mathrm{OCH}_{3}, 4-\mathrm{OCH}_{3}, 4-\mathrm{CH}_{3}, 4-\mathrm{NO}_{2}$ \\
\hline & $\mathrm{R}$ & 0.827 & 766.21 & 4.591 & 4.43 & 10 & $\mathrm{H}, 3-\mathrm{Br}, 2-\mathrm{Cl}, 4-\mathrm{Cl}, 4-\mathrm{F}, 4-\mathrm{OH}, 2-\mathrm{OCH}_{3}, 4-\mathrm{OCH}_{3}, 4-\mathrm{CH}_{3}, 4-\mathrm{NO}_{2}$ \\
\hline \multirow[t]{2}{*}{$\mathrm{CH}=\mathrm{CH}_{o p}$} & $\sigma$ & 0.907 & 1045.19 & 6.695 & 4.16 & 10 & $\mathrm{H}, 3-\mathrm{Br}, 2-\mathrm{Cl}, 4-\mathrm{Cl}, 4-\mathrm{F}, 4-\mathrm{OH}, 2-\mathrm{OCH}_{3}, 4-\mathrm{OCH}_{3}, 4-\mathrm{CH}_{3}, 4-\mathrm{NO}_{2}$ \\
\hline & $\sigma^{+}$ & 0.907 & 1048.88 & 8.885 & 4.17 & 10 & $\mathrm{H}, 3-\mathrm{Br}, 2-\mathrm{Cl}, 4-\mathrm{Cl}, 4-\mathrm{F}, 4-\mathrm{OH}, 2-\mathrm{OCH}_{3}, 4-\mathrm{OCH}_{3}, 4-\mathrm{CH}_{3}, 4-\mathrm{NO}_{2}$ \\
\hline
\end{tabular}

\begin{tabular}{|c|c|c|c|c|c|c|c|}
\hline & $\sigma_{1}$ & 0.847 & 1043.27 & 6.572 & 2.93 & 10 & $\mathrm{H}, 3-\mathrm{Br}, 2-\mathrm{Cl}, 4-\mathrm{Cl}, 4-\mathrm{F}, 4-\mathrm{OH}, 2-\mathrm{OCH}_{3}, 4-\mathrm{OCH}_{3}, 4-\mathrm{CH}_{3}, 4-\mathrm{NO}_{2}$ \\
\hline & $\sigma_{\mathrm{R}}$ & 0.906 & 1047.24 & 4.002 & 2.41 & 9 & $\mathrm{H}, 3-\mathrm{Br}, 2-\mathrm{Cl}, 4-\mathrm{Cl}, 4-\mathrm{F}, 4-\mathrm{OH}, 2-\mathrm{OCH}_{3}, 4-\mathrm{OCH}_{3}, 4-\mathrm{CH}_{3}$ \\
\hline & $\mathrm{F}$ & 0.830 & 1047.63 & 5.171 & 3.06 & 10 & $\mathrm{H}, 3-\mathrm{Br}, 2-\mathrm{Cl}, 4-\mathrm{Cl}, 4-\mathrm{F}, 4-\mathrm{OH}, 2-\mathrm{OCH}_{3}, 4-\mathrm{OCH}_{3}, 4-\mathrm{CH}_{3}, 4-\mathrm{NO}_{2}$ \\
\hline & $\mathrm{R}$ & 0.906 & 1047.84 & 7.542 & 2.47 & 9 & $\mathrm{H}, 3-\mathrm{Br}, 2-\mathrm{Cl}, 4-\mathrm{Cl}, 4-\mathrm{F}, 4-\mathrm{OH}, 2-\mathrm{OCH}_{3}, 4-\mathrm{OCH}_{3}, 4-\mathrm{CH}_{3}$ \\
\hline \multirow[t]{6}{*}{$\mathrm{C}=\mathrm{C}_{o p}$} & $\sigma$ & 0.905 & 570.25 & 7.409 & 4.19 & 8 & 3- $\mathrm{Br}, 2-\mathrm{Cl}, 4-\mathrm{Cl}, 4-\mathrm{F}, 2-\mathrm{OCH}_{3}, 4-\mathrm{OCH}_{3}, 4-\mathrm{CH}_{3}, 4-\mathrm{NO}_{2}$ \\
\hline & $\sigma^{+}$ & 0.905 & 570.98 & 4.794 & 4.27 & 8 & 3- $\mathrm{Br}, 2-\mathrm{Cl}, 4-\mathrm{Cl}, 4-\mathrm{F}, 2-\mathrm{OCH}_{3}, 4-\mathrm{OCH}_{3}, 4-\mathrm{CH}_{3}, 4-\mathrm{NO}_{2}$ \\
\hline & $\sigma_{1}$ & 0.823 & 568.68 & 8.741 & 4.91 & 10 & $\mathrm{H}, 3-\mathrm{Br}, 2-\mathrm{Cl}, 4-\mathrm{Cl}, 4-\mathrm{F}, 4-\mathrm{OH}, 2-\mathrm{OCH}_{3}, 4-\mathrm{OCH}_{3}, 4-\mathrm{CH}_{3}, 4-\mathrm{NO}_{2}$ \\
\hline & $\sigma_{\mathrm{R}}$ & 0.905 & 573.38 & 11.281 & 4.27 & 7 & $\mathrm{H}, 3-\mathrm{Br}, 2-\mathrm{Cl}, 4-\mathrm{Cl}, 4-\mathrm{OCH}_{3}, 4-\mathrm{CH}_{3}, 4-\mathrm{NO}_{2}$ \\
\hline & $\mathrm{F}$ & 0.827 & 568.60 & 5.546 & 4.90 & 10 & $\mathrm{H}, 3-\mathrm{Br}, 2-\mathrm{Cl}, 4-\mathrm{Cl}, 4-\mathrm{F}, 4-\mathrm{OH}, 2-\mathrm{OCH}_{3}, 4-\mathrm{OCH}_{3}, 4-\mathrm{CH}_{3}, 4-\mathrm{NO}_{2}$ \\
\hline & $\mathrm{R}$ & 0.905 & 573.29 & 8.643 & 4.41 & 7 & $\mathrm{H}, 3-\mathrm{Br}, 2-\mathrm{Cl}, 4-\mathrm{Cl}, 4-\mathrm{OCH}_{3}, 4-\mathrm{CH}_{3}, 4-\mathrm{NO}_{2}$ \\
\hline \multirow[t]{6}{*}{$\mathrm{NH}$} & $\sigma$ & 0.907 & 3321.03 & 27.485 & 9.78 & 10 & $\mathrm{H}, 3-\mathrm{Br}, 2-\mathrm{Cl}, 4-\mathrm{Cl}, 4-\mathrm{F}, 4-\mathrm{OH}, 2-\mathrm{OCH}_{3}, 4-\mathrm{OCH}_{3}, 4-\mathrm{CH}_{3}, 4-\mathrm{NO}_{2}$ \\
\hline & $\sigma^{+}$ & 0.906 & 3323.61 & 16.491 & 10.99 & 10 & $\mathrm{H}, 3-\mathrm{Br}, 2-\mathrm{Cl}, 4-\mathrm{Cl}, 4-\mathrm{F}, 4-\mathrm{OH}, 2-\mathrm{OCH}_{3}, 4-\mathrm{OCH}_{3}, 4-\mathrm{CH}_{3}, 4-\mathrm{NO}_{2}$ \\
\hline & $\sigma_{1}$ & 0.906 & 3309.27 & 38.483 & 11.33 & 9 & $\mathrm{H}, 3-\mathrm{Br}, 2-\mathrm{Cl}, 4-\mathrm{Cl}, 4-\mathrm{OH}, 2-\mathrm{OCH}_{3}, 4-\mathrm{OCH}_{3}, 4-\mathrm{CH}_{3}, 4-\mathrm{NO}_{2}$ \\
\hline & $\sigma_{\mathrm{R}}$ & 0.905 & 3329.68 & 29.895 & 12.55 & 8 & 3- $\mathrm{Br}, 2-\mathrm{Cl}, 4-\mathrm{Cl}, 4-\mathrm{F}, 4-\mathrm{OH}, 2-\mathrm{OCH}_{3}, 4-\mathrm{OCH}_{3}, 4-\mathrm{NO}_{2}$ \\
\hline & $\mathrm{F}$ & 0.843 & 3313.24 & 25.148 & 13.13 & 10 & $\mathrm{H}, 3-\mathrm{Br}, 2-\mathrm{Cl}, 4-\mathrm{Cl}, 4-\mathrm{F}, 4-\mathrm{OH}, 2-\mathrm{OCH}_{3}, 4-\mathrm{OCH}_{3}, 4-\mathrm{CH}_{3}, 4-\mathrm{NO}_{2}$ \\
\hline & $\mathrm{R}$ & 0.915 & 3330.85 & 27.462 & 12.11 & 8 & 3- $\mathrm{Br}, 2-\mathrm{Cl}, 4-\mathrm{Cl}, 4-\mathrm{F}, 4-\mathrm{OH}, 2-\mathrm{OCH}_{3}, 4-\mathrm{OCH}_{3}, 4-\mathrm{NO}_{2}$ \\
\hline \multirow[t]{6}{*}{$\mathrm{SO}_{2}$} & $\sigma$ & 0.833 & 1321.13 & 3.340 & 3.97 & 10 & $\mathrm{H}, 3-\mathrm{Br}, 2-\mathrm{Cl}, 4-\mathrm{Cl}, 4-\mathrm{F}, 4-\mathrm{OH}, 2-\mathrm{OCH}_{3}, 4-\mathrm{OCH}_{3}, 4-\mathrm{CH}_{3}, 4-\mathrm{NO}_{2}$ \\
\hline & $\sigma^{+}$ & 0.836 & 1321.15 & 2.656 & 3.91 & 10 & $\mathrm{H}, 3-\mathrm{Br}, 2-\mathrm{Cl}, 4-\mathrm{Cl}, 4-\mathrm{F}, 4-\mathrm{OH}, 2-\mathrm{OCH}_{3}, 4-\mathrm{OCH}_{3}, 4-\mathrm{CH}_{3}, 4-\mathrm{NO}_{2}$ \\
\hline & $\sigma_{1}$ & 0.811 & 1320.89 & 2.069 & 4.18 & 10 & $\mathrm{H}, 3-\mathrm{Br}, 2-\mathrm{Cl}, 4-\mathrm{Cl}, 4-\mathrm{F}, 4-\mathrm{OH}, 2-\mathrm{OCH}_{3}, 4-\mathrm{OCH}_{3}, 4-\mathrm{CH}_{3}, 4-\mathrm{NO}_{2}$ \\
\hline & $\sigma_{\mathrm{R}}$ & 0.836 & 1322.28 & 6.187 & 3.92 & 10 & $\mathrm{H}, 3-\mathrm{Br}, 2-\mathrm{Cl}, 4-\mathrm{Cl}, 4-\mathrm{F}, 4-\mathrm{OH}, 2-\mathrm{OCH}_{3}, 4-\mathrm{OCH}_{3}, 4-\mathrm{CH}_{3}, 4-\mathrm{NO}_{2}$ \\
\hline & $\mathrm{F}$ & 0.811 & 1320.59 & 1.954 & 4.18 & 10 & $\mathrm{H}, 3-\mathrm{Br}, 2-\mathrm{Cl}, 4-\mathrm{Cl}, 4-\mathrm{F}, 4-\mathrm{OH}, 2-\mathrm{OCH}_{3}, 4-\mathrm{OCH}_{3}, 4-\mathrm{CH}_{3}, 4-\mathrm{NO}_{2}$ \\
\hline & $\mathrm{R}$ & 0.841 & 1323.16 & 5.944 & 3.82 & 10 & $\mathrm{H}, 3-\mathrm{Br}, 2-\mathrm{Cl}, 4-\mathrm{Cl}, 4-\mathrm{F}, 4-\mathrm{OH}, 2-\mathrm{OCH}_{3}, 4-\mathrm{OCH}_{3}, 4-\mathrm{CH}_{3}, 4-\mathrm{NO}_{2}$ \\
\hline \multirow[t]{6}{*}{$\mathrm{CO}_{s y d}$} & $\sigma$ & 0.907 & 1755.64 & 21.382 & 7.65 & 8 & $\mathrm{H}, 3-\mathrm{Br}, 2-\mathrm{Cl}, 4-\mathrm{Cl}, 4-\mathrm{F}, 4-\mathrm{OCH}_{3}, 4-\mathrm{CH}_{3}, 4-\mathrm{NO}_{2}$ \\
\hline & $\sigma^{+}$ & 0.905 & 1757.52 & 11.630 & 9.12 & 8 & $\mathrm{H}, 3-\mathrm{Br}, 2-\mathrm{Cl}, 4-\mathrm{Cl}, 4-\mathrm{F}, 4-\mathrm{OCH}_{3}, 4-\mathrm{CH}_{3}, 4-\mathrm{NO}_{2}$ \\
\hline & $\sigma_{1}$ & 0.904 & 1749.03 & 22.330 & 10.04 & 8 & 3- $\mathrm{Br}, 2-\mathrm{Cl}, 4-\mathrm{Cl}, 4-\mathrm{F}, 4-\mathrm{OH}, 2-\mathrm{OCH}_{3}, 4-\mathrm{OCH}_{3}, 4-\mathrm{NO}_{2}$ \\
\hline & $\sigma_{\mathrm{R}}$ & 0.906 & 1763.85 & 29.361 & 8.72 & 8 & $\mathrm{H}, 3-\mathrm{Br}, 2-\mathrm{Cl}, 4-\mathrm{Cl}, 4-\mathrm{F}, 4-\mathrm{OCH}_{3}, 4-\mathrm{CH}_{3}, 4-\mathrm{NO}_{2}$ \\
\hline & $\mathrm{F}$ & 0.841 & 1749.92 & 18.535 & 10.37 & 10 & $\mathrm{H}, 3-\mathrm{Br}, 2-\mathrm{Cl}, 4-\mathrm{Cl}, 4-\mathrm{F}, 4-\mathrm{OH}, 2-\mathrm{OCH}_{3}, 4-\mathrm{OCH}_{3}, 4-\mathrm{CH}_{3}, 4-\mathrm{NO}_{2}$ \\
\hline & $\mathrm{R}$ & 0.905 & 1763.71 & 22.830 & 9.16 & 9 & $\mathrm{H}, 3-\mathrm{Br}, 2-\mathrm{Cl}, 4-\mathrm{Cl}, 4-\mathrm{F}, 2-\mathrm{OCH}_{3}, 4-\mathrm{OCH}_{3}, 4-\mathrm{CH}_{3}, 4-\mathrm{NO}_{2}$ \\
\hline
\end{tabular}


The correlation of deformation modes of $\mathrm{CH}_{i p}\left(v, \mathrm{~cm}^{-1}\right)$ of the synthesised sydnone chalcones gave satisfactory correlations with Hammett $\sigma, \sigma^{+}, \sigma_{I}$ constants and $\mathrm{F}$ parameters excluding $\mathrm{H}, 2-\mathrm{OCH}_{3}$ and $4-\mathrm{CH}_{3}$ substituents. The resonance components of the substituents were fail in correlation.

The correlation of deformation modes of $\mathrm{CH}_{o p}\left(v, \mathrm{~cm}^{-1}\right)$ of the synthesised sydnone chalcones gave satisfactory correlations with Hammett $\sigma, \sigma^{+}, \sigma_{I}$ constants and $F$ parameters excluding 4-F and 4-OH substituents. Also here the resonance components of the substituents were fail in correlation.

The correlation of deformation modes of $\mathrm{CH}=\mathrm{CH}_{o p}\left(v, \mathrm{~cm}^{-1}\right)$ of the synthesised sydnone chalcones gave satisfactory correlations with Hammett $\sigma, \sigma^{+}, \sigma_{\mathrm{R}}$ constants and $\mathrm{R}$ parameters excluding 4- $\mathrm{NO}_{2}$ substituent. The inductive and field components of the substituents were fail in correlation.

The correlation of deformation modes of $\mathrm{C}=\mathrm{C}_{o p}\left(v, \mathrm{~cm}^{-1}\right)$ of the synthesised sydnone chalcones gave satisfactory correlations with Hammett $\sigma, \sigma^{+}, \sigma_{\mathrm{R}}$ constants and $\mathrm{R}$ parameters excluding $\mathrm{H}, 4-\mathrm{F}, 4-\mathrm{OH}$ and $2-\mathrm{OCH}_{3}$ substituents. The inductive and field components of the substituents were fail in correlation.

The $\mathrm{NH}$ stretches $\left(v, \mathrm{~cm}^{-1}\right)$ of chalcones gave satisfactory correlation with Hammett substituent constants and $\mathrm{R}$ parameters excluding $4-\mathrm{F}$ and $4-\mathrm{CH}_{3}$ substituents. Here, the field components of the substituents were fail in correlation.

The poor correlations obtained for the $\mathrm{SO}_{2}$ stretches $\left(v, \mathrm{~cm}^{-1}\right)$ of chalcones with Hammett substituent constants, $\mathrm{F}$ and $\mathrm{R}$ parameters.

A satisfactory correlation was obtained for $\mathrm{CO}_{\text {syd }}\left(v, \mathrm{~cm}^{-1}\right)$ of chalcones with Hammett substituent constants and $\mathrm{R}$ parameters. The field effect of the substituents were fail for production of correlation. The reason for failure in correlation was already stated and along with resonanceconjugation structure as illustrated in Fig. 2.

In single parameter infrared spectral correlations, some of the Hammett substituent constants, $\mathrm{F}$ and $\mathrm{R}$ parameters were fail for giving correlation coefficients. When these data are produced satisfactory correlations in multi-regression analysis with $\sigma_{\mathrm{I}}, \sigma_{\mathrm{R}}$ and Swain-Lupton's constants[33]. The generated multi-regression analysis equations are given in (2-19).

$$
\begin{aligned}
& v \mathrm{CO}_{s-c i s}\left(\mathrm{~cm}^{-1}\right)=1661.14( \pm 5.453)+43.603( \pm 11.700) \sigma_{\mathrm{I}}+65.843( \pm 11.277) \sigma_{\mathrm{R}} \\
& (R=0.983, \quad \mathrm{n}=10, \quad \mathrm{P}>95 \%) \\
& v \mathrm{CO}_{s-c i s}\left(\mathrm{~cm}^{-1}\right)=1660.04( \pm 6.327)+40.784( \pm 13.298) \mathrm{F}+49.056( \pm 11.336) \mathrm{R} \\
& (R=0.990, \quad \mathrm{n}=10, \quad \mathrm{P}>95 \%) \\
& \nu \mathrm{CO}_{\text {s-trans }}\left(\mathrm{cm}^{-1}\right)=1606.83( \pm 3.0517)+1.749( \pm 0.124) \sigma_{\mathrm{I}}+30.863( \pm 6.314) \sigma_{\mathrm{R}} \\
& (R=0.987, \quad \mathrm{n}=10, \quad \mathrm{P}>95 \%) \\
& v \mathrm{CO}_{\text {s-trans }}\left(\mathrm{cm}^{-1}\right)=1606.45( \pm 3.322)+3.268( \pm 0.691) \mathrm{F}+25.185( \pm 5.953) \mathrm{R} \\
& (R=0.990, \quad \mathrm{n}=10, \quad \mathrm{P}>95 \%) \\
& v \mathrm{CH}_{i p}\left(\mathrm{~cm}^{-1}\right)=1168.76( \pm 2.737)+20.085( \pm 5.959) \sigma_{\mathrm{I}}+5.139( \pm 5.749) \sigma_{\mathrm{R}} \\
& (R=0.973, \quad \mathrm{n}=10, \quad \mathrm{P}>95 \%) \\
& v \mathrm{CH}_{i p}\left(\mathrm{~cm}^{-1}\right)=1169.79( \pm 2.736)+18.745( \pm 5.780) \mathrm{F}+2.236( \pm 4.903) \mathrm{R} \\
& (R=0.979, \quad \mathrm{n}=10, \quad \mathrm{P}>95 \%) \\
& v \mathrm{CH}_{o p}\left(\mathrm{~cm}^{-1}\right)=761.50( \pm 2.109)+13.995( \pm 4.526) \sigma_{\mathrm{I}}+5.884( \pm 2.314) \sigma_{\mathrm{R}} \\
& (R=0.978, \quad \mathrm{n}=10, \quad \mathrm{P}>95 \%) \\
& v \mathrm{CH}_{o p}\left(\mathrm{~cm}^{-1}\right)=762.24( \pm 2.334)+12.107( \pm 4.905) \mathrm{F}+5.814( \pm 2.118) \mathrm{R} \\
& (R=0.971, \quad \mathrm{n}=10, \quad \mathrm{P}>95 \%) \\
& v \mathrm{CH}=\mathrm{CH}_{o p}\left(\mathrm{~cm}^{-1}\right)=1045.49( \pm 1.406)+6.574( \pm 3.014) \sigma_{\mathrm{I}}+9.020( \pm 2.910) \sigma_{\mathrm{R}} \\
& (R=0.981, \quad \mathrm{n}=10, \quad \mathrm{P}>95 \%) \\
& v \mathrm{CH}=\mathrm{CH}_{o p}\left(\mathrm{~cm}^{-1}\right)=1045.76( \pm 1.364)+6.352( \pm 2.867) \mathrm{F}+8.207( \pm 2.445) \mathrm{R} \\
& (R=0.982, \quad \mathrm{n}=10, \quad \mathrm{P}>95 \%) \\
& \nu \mathrm{C}=\mathrm{C}_{o p}\left(\mathrm{~cm}^{-1}\right)=571.44( \pm 2.988)+5.740( \pm 0.625) \sigma_{\mathrm{I}}+11.280( \pm 5.210) \sigma_{\mathrm{R}} \\
& (R=0.960, \quad \mathrm{n}=10, \quad \mathrm{P}>95 \%)
\end{aligned}
$$




$$
\begin{aligned}
& v \mathrm{C}=\mathrm{C}_{o p}\left(\mathrm{~cm}^{-1}\right)=571.03( \pm 2.931)+6.895( \pm 1.625) \mathrm{F}+9.370( \pm 2.697) \mathrm{R} \\
& (R=0.960, \quad \mathrm{n}=10, \quad \mathrm{P}>95 \%) \\
& v \mathrm{NH}\left(\mathrm{cm}^{-1}\right)=3316.65( \pm 6.331)+38.429( \pm 13.888) \sigma_{\mathrm{I}}+29.893( \pm 13.098) \sigma_{\mathrm{R}} \\
& (R=0.980, \quad \mathrm{n}=10, \quad \mathrm{P}>95 \%) \\
& v \mathrm{NH}\left(\mathrm{cm}^{-1}\right)=3321.16( \pm 6.921)+29.544( \pm 4.258) \mathrm{F}+30.554( \pm 5.368) \mathrm{R} \\
& (R=0.975, \mathrm{n}=10, \mathrm{P}>95 \%) \\
& v \mathrm{SO}\left(\mathrm{cm}^{-1}\right)=1322.12( \pm 2.875)+2.068( \pm 6.169) \sigma_{\mathrm{I}}+6.180( \pm 1.325) \sigma_{\mathrm{R}} \\
& (R=0.938, \quad \mathrm{n}=10, \quad \mathrm{P}>90 \%) \\
& v \mathrm{SO}\left(\mathrm{cm}^{-1}\right)=1322.17( \pm 2.706)+12.895( \pm 5.685) \mathrm{F}+6.288( \pm 1.850) \mathrm{R} \\
& (R=0.945, \quad \mathrm{n}=10, \quad \mathrm{P}>90 \%) \\
& v \mathrm{CO}_{\text {syd }}\left(\mathrm{cm}^{-1}\right)=1756.28( \pm 5.144)+22.329( \pm 1.025) \sigma_{\mathrm{I}}+29.365( \pm 10.571) \sigma_{\mathrm{R}} \\
& (R=0.979, \quad \mathrm{n}=10, \quad \mathrm{P}>95 \%) \\
& v \mathrm{CO}_{\text {syd }}\left(\mathrm{cm}^{-1}\right)=1756.44( \pm 5.256)+22.154( \pm 6.358) \mathrm{F}+25.148( \pm 0.942) \mathrm{R} \\
& (R=0.976, \mathrm{n}=10, \quad \mathrm{P}>90 \%)
\end{aligned}
$$

\subsection{NMR Spectral correlations}

\subsection{1. ${ }^{1} \mathrm{H}$ NMR spectral study}

In the present study, the compounds chosen for evaluating the effects of substituents on the sydnone chalcones was shown in Fig. 1. The NMR spectra of synthesised sydnone chalcone were recorded in $\mathrm{CDCl}_{3}$ solvent using TMS as internal standard. The Chemical shifts $(\delta, \mathrm{ppm})$ of vinyl protons, $\mathrm{NH}$ and methyl protons of synthesised sydnone chalcones were presented in Table 3 . These chemical shifts $(\delta$, ppm) have been correlated with Hammett substituent constants, F and R parameters using single and multi-linear regression analysis [13-16, 20-32]. In this correlation the Hammett equation was taken in the form as(20)

$$
\delta=\rho \sigma+\delta_{\mathrm{o}}
$$

where $\delta_{\mathrm{o}}$ is the frequency for the parent member of the series.

Table 3. The ${ }^{1} \mathrm{H}$ NMR chemical shifts $(\delta, \mathrm{ppm})$ of $(E)-4-(N-(4-\operatorname{cinnamoylphenyl)~sulfamoyl)-3-(p-}$ tolyl)-1,2,3-oxadiazol-3-ium-5-olates.

\begin{tabular}{|l|l|l|l|l|l|}
\hline Entry & $\mathrm{X}$ & $\mathrm{H}_{\alpha}$ & $\mathrm{H}_{\beta}$ & $\mathrm{NH}$ & $\mathrm{CH}_{3}$ \\
\hline 1 & $\mathrm{H}$ & 6.853 & 7.431 & 8.243 & 2.381 \\
\hline 2 & $3-\mathrm{Br}$ & 6.781 & 7.452 & 8.217 & 2.343 \\
\hline 3 & $2-\mathrm{Cl}$ & 6.281 & 7.603 & 9.213 & 2.317 \\
\hline 4 & $4-\mathrm{Cl}$ & 7.411 & 6.413 & 9.413 & 2.372 \\
\hline 5 & $4-\mathrm{F}$ & 7.613 & 8.171 & 9.571 & 2.345 \\
\hline 6 & $4-\mathrm{OH}$ & 7.061 & 7.123 & 9.459 & 2.460 \\
\hline 7 & $2-\mathrm{OCH}_{3}$ & 7.071 & 7.172 & 9.381 & 2.361 \\
\hline 8 & $4-\mathrm{OCH}_{3}$ & 6.671 & 7.342 & 9.109 & 2.306 \\
\hline 9 & $4-\mathrm{CH}_{3}$ & 6.687 & 7.347 & 9.061 & 2.297 \\
\hline 10 & $4-\mathrm{NO}_{2}$ & 7.773 & 7.643 & 9.721 & 2.413 \\
\hline
\end{tabular}

The results of statistical analysis of these proton chemical shifts of sydnone chalcones with Hammett substituent constants were presented in Table 4. 
Table 4. Results of statistical analysis of ${ }^{1} \mathrm{H}$ NMR chemical shifts $(\delta$, ppm) of $(E)-4-(N-(4-$ cinnamoylphenyl)sulfamoyl)-3-( $p$-tolyl)-1,2,3-oxadiazol-3-ium-5-olates with Hammett substituent constants, F and R parameters.

\begin{tabular}{|c|c|c|c|c|c|c|c|}
\hline Frequency & Constant & $\mathrm{r}$ & I & $\rho$ & $\mathrm{s}$ & $\mathrm{n}$ & Correlated derivatives \\
\hline \multirow[t]{6}{*}{$\mathrm{H}_{\alpha}$} & $\sigma$ & 0.837 & 6.907 & 0.491 & 0.15 & 10 & $\mathrm{H}, 3-\mathrm{Br}, 2-\mathrm{Cl}, 4-\mathrm{Cl}, 4-\mathrm{F}, 4-\mathrm{OH}, 2-\mathrm{OCH}_{3}, 4-\mathrm{OCH}_{3}, 4-\mathrm{CH}_{3}, 4-\mathrm{NO}_{2}$ \\
\hline & $\sigma^{+}$ & 0.819 & 7.006 & 0.165 & 0.15 & 10 & $\mathrm{H}, 3-\mathrm{Br}, 2-\mathrm{Cl}, 4-\mathrm{Cl}, 4-\mathrm{F}, 4-\mathrm{OH}, 2-\mathrm{OCH}_{3}, 4-\mathrm{OCH}_{3}, 4-\mathrm{CH}_{3}, 4-\mathrm{NO}_{2}$ \\
\hline & $\sigma_{1}$ & 0.905 & 6.572 & 1.242 & 0.11 & 8 & $\mathrm{H}, 4-\mathrm{Cl}, 4-\mathrm{F}, 4-\mathrm{OH}, 2-\mathrm{OCH}_{3}, 4-\mathrm{OCH}_{3}, 4-\mathrm{CH}_{3}, 4-\mathrm{NO}_{2}$ \\
\hline & $\sigma_{\mathrm{R}}$ & 0.804 & 7.001 & 0.096 & 0.16 & 10 & $\mathrm{H}, 3-\mathrm{Br}, 2-\mathrm{Cl}, 4-\mathrm{Cl}, 4-\mathrm{F}, 4-\mathrm{OH}, 2-\mathrm{OCH}_{3}, 4-\mathrm{OCH}_{3}, 4-\mathrm{CH}_{3}, 4-\mathrm{NO}_{2}$ \\
\hline & $\mathrm{F}$ & 0.906 & 6.499 & 1.347 & 0.12 & 8 & $\mathrm{H}, 4-\mathrm{Cl}, 4-\mathrm{F}, 4-\mathrm{OH}, 2-\mathrm{OCH}_{3}, 4-\mathrm{OCH}_{3}, 4-\mathrm{CH}_{3}, 4-\mathrm{NO}_{2}$ \\
\hline & $\mathrm{R}$ & 0.800 & 6.995 & 0.807 & 0.16 & 10 & $\mathrm{H}, 3-\mathrm{Br}, 2-\mathrm{Cl}, 4-\mathrm{Cl}, 4-\mathrm{F}, 4-\mathrm{OH}, 2-\mathrm{OCH}_{3}, 4-\mathrm{OCH}_{3}, 4-\mathrm{CH}_{3}, 4-\mathrm{NO}_{2}$ \\
\hline \multirow[t]{6}{*}{$\mathrm{H}_{\beta}$} & $\sigma$ & 0.907 & 7.372 & 0.408 & 0.12 & 10 & $\mathrm{H}, 3-\mathrm{Br}, 2-\mathrm{Cl}, 4-\mathrm{Cl}, 4-\mathrm{F}, 4-\mathrm{OH}, 2-\mathrm{OCH}_{3}, 4-\mathrm{OCH}_{3}, 4-\mathrm{CH}_{3}, 4-\mathrm{NO}_{2}$ \\
\hline & $\sigma^{+}$ & 0.908 & 7.414 & 0.279 & 0.12 & 10 & $\mathrm{H}, 3-\mathrm{Br}, 2-\mathrm{Cl}, 4-\mathrm{Cl}, 4-\mathrm{F}, 4-\mathrm{OH}, 2-\mathrm{OCH}_{3}, 4-\mathrm{OCH}_{3}, 4-\mathrm{CH}_{3}, 4-\mathrm{NO}_{2}$ \\
\hline & $\sigma_{\mathrm{I}}$ & 0.834 & 7.792 & 0.292 & 0.19 & 10 & $\mathrm{H}, 3-\mathrm{Br}, 2-\mathrm{Cl}, 4-\mathrm{Cl}, 4-\mathrm{F}, 4-\mathrm{OH}, 2-\mathrm{OCH}_{3}, 4-\mathrm{OCH}_{3}, 4-\mathrm{CH}_{3}, 4-\mathrm{NO}_{2}$ \\
\hline & $\sigma_{\mathrm{R}}$ & 0.907 & 7.542 & 0.626 & 0.13 & 10 & $\mathrm{H}, 3-\mathrm{Br}, 2-\mathrm{Cl}, 4-\mathrm{Cl}, 4-\mathrm{F}, 4-\mathrm{OH}, 2-\mathrm{OCH}_{3}, 4-\mathrm{OCH}_{3}, 4-\mathrm{CH}_{3}, 4-\mathrm{NO}_{2}$ \\
\hline & $\mathrm{F}$ & 0.813 & 7.358 & 0.104 & 0.13 & 10 & $\mathrm{H}, 3-\mathrm{Br}, 2-\mathrm{Cl}, 4-\mathrm{Cl}, 4-\mathrm{F}, 4-\mathrm{OH}, 2-\mathrm{OCH}_{3}, 4-\mathrm{OCH}_{3}, 4-\mathrm{CH}_{3}, 4-\mathrm{NO}_{2}$ \\
\hline & $\mathrm{R}$ & 0.908 & 7.568 & 0.573 & 0.11 & 10 & $\mathrm{H}, 3-\mathrm{Br}, 2-\mathrm{Cl}, 4-\mathrm{Cl}, 4-\mathrm{F}, 4-\mathrm{OH}, 2-\mathrm{OCH}_{3}, 4-\mathrm{OCH}_{3}, 4-\mathrm{CH}_{3}, 4-\mathrm{NO}_{2}$ \\
\hline \multirow[t]{6}{*}{ NH } & $\sigma$ & 0.801 & 9.130 & 0.140 & 0.54 & 10 & $\mathrm{H}, 3-\mathrm{Br}, 2-\mathrm{Cl}, 4-\mathrm{Cl}, 4-\mathrm{F}, 4-\mathrm{OH}, 2-\mathrm{OCH}_{3}, 4-\mathrm{OCH}_{3}, 4-\mathrm{CH}_{3}, 4-\mathrm{NO}_{2}$ \\
\hline & $\sigma^{+}$ & 0.809 & 9.132 & 0.084 & 0.54 & 10 & $\mathrm{H}, 3-\mathrm{Br}, 2-\mathrm{Cl}, 4-\mathrm{Cl}, 4-\mathrm{F}, 4-\mathrm{OH}, 2-\mathrm{OCH}_{3}, 4-\mathrm{OCH}_{3}, 4-\mathrm{CH}_{3}, 4-\mathrm{NO}_{2}$ \\
\hline & $\sigma_{\mathrm{l}}$ & 0.904 & 8.723 & 1.088 & 0.48 & 9 & $\mathrm{H}, 2-\mathrm{Cl}, 4-\mathrm{Cl}, 4-\mathrm{F}, 4-\mathrm{OH}, 2-\mathrm{OCH}_{3}, 4-\mathrm{OCH}_{3}, 4-\mathrm{CH}_{3}, 4-\mathrm{NO}_{2}$ \\
\hline & $\sigma_{\mathrm{R}}$ & 0.828 & 8.989 & 0.639 & 0.52 & 10 & $\mathrm{H}, 3-\mathrm{Br}, 2-\mathrm{Cl}, 4-\mathrm{Cl}, 4-\mathrm{F}, 4-\mathrm{OH}, 2-\mathrm{OCH}_{3}, 4-\mathrm{OCH}_{3}, 4-\mathrm{CH}_{3}, 4-\mathrm{NO}_{2}$ \\
\hline & $\mathrm{F}$ & 0.905 & 8.174 & 1.181 & 0.46 & 8 & $2-\mathrm{Cl}, 4-\mathrm{Cl}, 4-\mathrm{F}, 4-\mathrm{OH}, 2-\mathrm{OCH}_{3}, 4-\mathrm{OCH}_{3}, 4-\mathrm{CH}_{3}, 4-\mathrm{NO}_{2}$ \\
\hline & $\mathrm{R}$ & 0.828 & 8.971 & 0.538 & 0.52 & 10 & $\mathrm{H}, 3-\mathrm{Br}, 2-\mathrm{Cl}, 4-\mathrm{Cl}, 4-\mathrm{F}, 4-\mathrm{OH}, 2-\mathrm{OCH}_{3}, 4-\mathrm{OCH}_{3}, 4-\mathrm{CH}_{3}, 4-\mathrm{NO}_{2}$ \\
\hline \multirow[t]{6}{*}{$\mathrm{CH}_{3}$} & $\sigma$ & 0.817 & 2.358 & 0.014 & 0.05 & 10 & $\mathrm{H}, 3-\mathrm{Br}, 2-\mathrm{Cl}, 4-\mathrm{Cl}, 4-\mathrm{F}, 4-\mathrm{OH}, 2-\mathrm{OCH}_{3}, 4-\mathrm{OCH}_{3}, 4-\mathrm{CH}_{3}, 4-\mathrm{NO}_{2}$ \\
\hline & $\sigma^{+}$ & 0.805 & 2.359 & 0.047 & 0.05 & 10 & $\mathrm{H}, 3-\mathrm{Br}, 2-\mathrm{Cl}, 4-\mathrm{Cl}, 4-\mathrm{F}, 4-\mathrm{OH}, 2-\mathrm{OCH}_{3}, 4-\mathrm{OCH}_{3}, 4-\mathrm{CH}_{3}, 4-\mathrm{NO}_{2}$ \\
\hline & $\sigma_{1}$ & 0.820 & 2.344 & 0.441 & 0.05 & 10 & $\mathrm{H}, 3-\mathrm{Br}, 2-\mathrm{Cl}, 4-\mathrm{Cl}, 4-\mathrm{F}, 4-\mathrm{OH}, 2-\mathrm{OCH}_{3}, 4-\mathrm{OCH}_{3}, 4-\mathrm{CH}_{3}, 4-\mathrm{NO}_{2}$ \\
\hline & $\sigma_{R}$ & 0.809 & 2.364 & 0.024 & 0.05 & 10 & $\mathrm{H}, 3-\mathrm{Br}, 2-\mathrm{Cl}, 4-\mathrm{Cl}, 4-\mathrm{F}, 4-\mathrm{OH}, 2-\mathrm{OCH}_{3}, 4-\mathrm{OCH}_{3}, 4-\mathrm{CH}_{3}, 4-\mathrm{NO}_{2}$ \\
\hline & $\mathrm{F}$ & 0.820 & 2.344 & 0.042 & 0.05 & 10 & $\mathrm{H}, 3-\mathrm{Br}, 2-\mathrm{Cl}, 4-\mathrm{Cl}, 4-\mathrm{F}, 4-\mathrm{OH}, 2-\mathrm{OCH}_{3}, 4-\mathrm{OCH}_{3}, 4-\mathrm{CH}_{3}, 4-\mathrm{NO}_{2}$ \\
\hline & $\mathrm{R}$ & 0.826 & 2.358 & 0.041 & 0.05 & 10 & $\mathrm{H}, 3-\mathrm{Br}, 2-\mathrm{Cl}, 4-\mathrm{Cl}, 4-\mathrm{F}, 4-\mathrm{OH}, 2-\mathrm{OCH}_{3}, 4-\mathrm{OCH}_{3}, 4-\mathrm{CH}_{3}, 4-\mathrm{NO}_{2}$ \\
\hline
\end{tabular}

From Table 4, the correlation of $\mathrm{H}_{\alpha}$ chemical shifts (ppm) of sydnone chalcones gave satisfactory correlation coefficients with Hammett $\sigma_{\mathrm{I}}$ constant and $\mathrm{F}$ parameter excluding 3-Br and 2-Cl substituents. The remaining Hammett $\sigma, \sigma^{+}, \sigma_{\mathrm{R}}$ constants and $\mathrm{R}$ parameters were failing in correlations.

A satisfactory correlation was obtained for $\mathrm{H}_{\beta}$ chemical shifts (ppm) with Hammett $\sigma, \sigma^{+}$, $\sigma_{R}$ constants and R parameters. The inductive and field components of the substituents were fail in correlations.

The Hammett $\sigma_{\mathrm{I}}$ constant and $\mathrm{F}$ parameters gave satisfactory correlation with the chemical shifts(ppm) of NH protons of the synthesised sydnone chalcones excluding 3-Br substituent. The Hammett $\sigma, \sigma^{+}, \sigma_{\mathrm{R}}$ constants and R parameters gave poor correlations with $\mathrm{NH}$ proton chemical shifts(ppm) of sydnone chalcones,

The Hammett substituent constants, $\mathrm{F}$ and $\mathrm{R}$ parameters were fail in correlation for the methyl proton chemical shifts(ppm) of sydnone chalcones. The reason for the failure in correlation was already stated in earlier and associated with resonance-conjugated structure as shown in Fig. 2. In proton chemical shift correlations, all single regressions gave positive $\rho$ values. This means that the normal substituent effect operates in all system.

In single parameter proton chemical shift correlations, some of them are failed. While seeking these in multi-regression analysis[33], they are worthwhile and produced satisfactory correlations. The generated multi-regression analysis equations are given in(21-28).

$$
\begin{gathered}
\delta \mathrm{H}_{\alpha}(\mathrm{ppm})=6.595( \pm 0.307)+1.242( \pm 0.655) \sigma_{\mathrm{I}}+0.990( \pm 0.065) \sigma_{\mathrm{R}} \\
(R=0.958, \mathrm{n}=10, \quad \mathrm{P}>95 \%) \\
\delta \mathrm{H}_{\alpha}(\mathrm{ppm})=6.539( \pm 0.270)+1.393( \pm 0.567) \mathrm{F}+0.153( \pm 0.045) \mathrm{R} \\
\quad(R=0.968, \mathrm{n}=10, \mathrm{P}>95 \%) \\
\delta \mathrm{H}_{\beta}(\mathrm{ppm})=7.447( \pm 0.081)+0.292( \pm 0.174) \sigma_{\mathrm{I}}+0.620( \pm 0.124) \sigma_{\mathrm{R}}
\end{gathered}
$$




$$
\begin{aligned}
&(R=0.983, \mathrm{n}=10, \quad \mathrm{P}>95 \%) \\
& \delta \mathrm{H}_{\beta}(\mathrm{ppm})=7.505( \pm 0.073)+0.192( \pm 0.012) \mathrm{F}+0.568( \pm 0.125) \mathrm{R} \\
&(R=0.986, \mathrm{n}=10, \quad \mathrm{P}>95 \%) \\
& \delta \mathrm{NH}(\mathrm{ppm})=8.614( \pm 1.089)+1.085( \pm 0.732) \sigma_{\mathrm{I}}+0.630( \pm 0.074) \sigma_{\mathrm{R}}(R=0.959, \mathrm{n}=10, \quad \mathrm{P}>95 \%) \\
& \delta \mathrm{NH}(\mathrm{ppm})=8.604( \pm 0.322)+1.122( \pm 0.625) \mathrm{F}+0.427( \pm 0.025) \mathrm{R} \\
& \\
&(R=0.958, \mathrm{n}=10, \quad \mathrm{P}>95 \%) \\
& \delta \mathrm{CH}_{3}(\mathrm{ppm})=2.349( \pm 0.036)+0.042( \pm 0.002) \sigma_{\mathrm{I}}+0.258( \pm 0.123) \sigma_{\mathrm{R}} \\
& \\
&(R=0.922, \mathrm{n}=10, \quad \mathrm{P}>90 \%) \\
& \delta \mathrm{CH}_{3}(\mathrm{ppm})=2.344( \pm 0.037)+0.042( \pm 0.007) \mathrm{F}+0.303( \pm 0.067) \mathrm{R} \\
&(R=0.920, \mathrm{n}=10, \quad \mathrm{P}>90 \%)
\end{aligned}
$$

\subsection{2. ${ }^{13} \mathrm{C}$ NMR spectral study}

The ${ }^{13} \mathrm{C}$ NMR spectral chemical shifts(ppm) of synthesised sydnone chalcones are presented and Table 5. These data are correlated with Hammett substituent constants, $\mathrm{F}$ and $\mathrm{R}$ parameters using single and multi-linear regression analysis[13-16, 20-320]. The results of single parameter correlation analysis of ${ }^{13} \mathrm{C}$ NMR spectral chemical shifts(ppm) of synthesised sydnone chalcones are tabulated in Table 6. From Table 6, the correlation of $\delta \mathrm{CO}(\mathrm{ppm})$ of the chalcones gave satisfactory correlation coefficients with Hammett $\sigma^{+}, \sigma_{I}$ constants and F parameters. The remaining Hammett $\sigma, \sigma_{\mathrm{R}}$ constants and $\mathrm{R}$ parameters fail in correlations. This is due to the inability of substituents effects already stated and associated with conjugative structure as shown in Fig. 2.

Table 5. The ${ }^{13} \mathrm{C}$ NMR chemical shifts $(\delta, \mathrm{ppm})$ of $(E)-4-(N-(4-$ cinnamoylphenyl)sulfamoyl)-3- $(p-$ tolyl)-1,2,3-oxadiazol-3-ium-5-olates.

\begin{tabular}{|l|l|l|l|l|l|l|l|l|l|l|l|l|}
\hline Entry & $\mathrm{X}$ & $\mathrm{CO}$ & $\mathrm{C}_{a}$ & $\mathrm{C}_{\beta}$ & $\mathrm{Ar}-\mathrm{C}-\mathrm{C}_{\beta}$ & $\mathrm{Ar}-\mathrm{C}-\mathrm{CO}$ & $\mathrm{Ar}-\mathrm{C}-\mathrm{NH}$ & $\mathrm{C}-\mathrm{S}$ & $\mathrm{CO}_{s y d}$ & $\mathrm{Ar}-\mathrm{C}-\mathrm{N}^{+}$ & $\mathrm{Ar}_{-} \mathrm{C}-\mathrm{CH}_{3}$ & $\mathrm{CH}_{3}$ \\
\hline 1 & $\mathrm{H}$ & 188.74 & 119.23 & 145.27 & 127.52 & 127.66 & 144.72 & 110.28 & 172.74 & 135.82 & 141.73 & 23.31 \\
\hline 2 & $3-\mathrm{Br}$ & 188.69 & 119.24 & 140.38 & 127.54 & 127.28 & 144.36 & 110.34 & 174.13 & 135.71 & 141.28 & 22.52 \\
\hline 3 & $2-\mathrm{Cl}$ & 189.06 & 119.23 & 145.64 & 128.65 & 127.28 & 144.21 & 111.34 & 174.89 & 136.06 & 141.36 & 22.33 \\
\hline 4 & $4-\mathrm{Cl}$ & 188.85 & 120.13 & 145.37 & 128.35 & 127.14 & 144.28 & 110.21 & 175.24 & 135.68 & 141.31 & 22.87 \\
\hline 5 & $4-\mathrm{F}$ & 189.41 & 120.03 & 145.26 & 128.63 & 127.24 & 144.31 & 110.27 & 175.93 & 135.51 & 141.48 & 21.36 \\
\hline 6 & $4-\mathrm{OH}$ & 188.11 & 120.04 & 144.97 & 127.74 & 127.16 & 144.32 & 110.24 & 174.88 & 135.38 & 141.29 & 21.61 \\
\hline 7 & $2-\mathrm{OCH}$ & 188.96 & 120.14 & 145.19 & 127.36 & 127.01 & 144.14 & 110.19 & 174.92 & 138.27 & 141.21 & 21.35 \\
\hline 8 & $4-\mathrm{OCH}_{3}$ & 184.03 & 118.97 & 145.06 & 127.23 & 126.98 & 144.03 & 109.89 & 173.98 & 135.27 & 140.83 & 21.43 \\
\hline 9 & $4-\mathrm{CH}_{3}$ & 184.95 & 119.72 & 145.17 & 128.54 & 127.20 & 144.19 & 110.14 & 174.25 & 135.72 & 140.98 & 22.06 \\
\hline 10 & $4-\mathrm{NO}_{2}$ & 188.98 & 120.31 & 146.08 & 128.96 & 128.11 & 145.07 & 111.76 & 175.06 & 135.97 & 141.33 & 23.65 \\
\hline
\end{tabular}


Table 6. Results of statistical analysis of ${ }^{13} \mathrm{C}$ NMR chemical shifts $(\delta$, ppm) of $(E)-4-(N-(4-$ cinnamoylphenyl)sulfamoyl)-3-( $p$-tolyl)-1,2,3-oxadiazol-3-ium-5-olates with Hammett substituent constants, $\mathrm{F}$ and $\mathrm{R}$ parameters.

\begin{tabular}{|c|c|c|c|c|c|c|c|}
\hline Frequency & Constant & $\mathrm{r}$ & I & $\rho$ & $\mathrm{s}$ & $\mathrm{n}$ & Correlated derivatives \\
\hline \multirow[t]{6}{*}{$\mathrm{CO}$} & $\sigma$ & 0.848 & 188.62 & 0.673 & 0.48 & 10 & $\mathrm{H}, 3-\mathrm{Br}, 2-\mathrm{Cl}, 4-\mathrm{Cl}, 4-\mathrm{F}, 4-\mathrm{OH}, 2-\mathrm{OCH}_{3}, 4-\mathrm{OCH}_{3}, 4-\mathrm{CH}_{3}, 4-\mathrm{NO}_{2}$ \\
\hline & $\sigma^{+}$ & 0.906 & 188.70 & 0.883 & 0.43 & 10 & $\mathrm{H}, 3-\mathrm{Br}, 2-\mathrm{Cl}, 4-\mathrm{Cl}, 4-\mathrm{F}, 4-\mathrm{OH}, 2-\mathrm{OCH}_{3}, 4-\mathrm{OCH}_{3}, 4-\mathrm{CH}_{3}, 4-\mathrm{NO}_{2}$ \\
\hline & $\sigma_{l}$ & 0.906 & 188.16 & 1.430 & 0.43 & 10 & $\mathrm{H}, 3-\mathrm{Br}, 2-\mathrm{Cl}, 4-\mathrm{Cl}, 4-\mathrm{F}, 4-\mathrm{OH}, 2-\mathrm{OCH}_{3}, 4-\mathrm{OCH}_{3}, 4-\mathrm{CH}_{3}, 4-\mathrm{NO}_{2}$ \\
\hline & $\sigma_{\mathrm{R}}$ & 0.801 & 188.65 & 0.022 & 0.35 & 10 & $\mathrm{H}, 3-\mathrm{Br}, 2-\mathrm{Cl}, 4-\mathrm{Cl}, 4-\mathrm{F}, 4-\mathrm{OH}, 2-\mathrm{OCH}_{3}, 4-\mathrm{OCH}_{3}, 4-\mathrm{CH}_{3}, 4-\mathrm{NO}_{2}$ \\
\hline & $\mathrm{F}$ & 0.900 & 188.165 & 1.345 & 0.53 & 10 & $\mathrm{H}, 3-\mathrm{Br}, 2-\mathrm{Cl}, 4-\mathrm{Cl}, 4-\mathrm{F}, 4-\mathrm{OH}, 2-\mathrm{OCH}_{3}, 4-\mathrm{OCH}_{3}, 4-\mathrm{CH}_{3}, 4-\mathrm{NO}_{2}$ \\
\hline & $\mathrm{R}$ & 0.878 & 188.751 & 0.330 & 0.54 & 10 & $\mathrm{H}, 3-\mathrm{Br}, 2-\mathrm{Cl}, 4-\mathrm{Cl}, 4-\mathrm{F}, 4-\mathrm{OH}, 2-\mathrm{OCH}_{3}, 4-\mathrm{OCH}_{3}, 4-\mathrm{CH}_{3}, 4-\mathrm{NO}_{2}$ \\
\hline \multirow[t]{6}{*}{$\mathrm{C}_{\alpha}$} & $\sigma$ & 0.808 & 119.72 & 0.188 & 0.53 & 10 & $\mathrm{H}, 3-\mathrm{Br}, 2-\mathrm{Cl}, 4-\mathrm{Cl}, 4-\mathrm{F}, 4-\mathrm{OH}, 2-\mathrm{OCH}_{3}, 4-\mathrm{OCH}_{3}, 4-\mathrm{CH}_{3}, 4-\mathrm{NO}_{2}$ \\
\hline & $\sigma^{+}$ & 0.805 & 119.72 & 0.055 & 0.53 & 10 & $\mathrm{H}, 3-\mathrm{Br}, 2-\mathrm{Cl}, 4-\mathrm{Cl}, 4-\mathrm{F}, 4-\mathrm{OH}, 2-\mathrm{OCH}_{3}, 4-\mathrm{OCH}_{3}, 4-\mathrm{CH}_{3}, 4-\mathrm{NO}_{2}$ \\
\hline & $\sigma_{1}$ & 0.832 & 119.47 & 0.732 & 0.51 & 10 & $\mathrm{H}, 3-\mathrm{Br}, 2-\mathrm{Cl}, 4-\mathrm{Cl}, 4-\mathrm{F}, 4-\mathrm{OH}, 2-\mathrm{OCH}_{3}, 4-\mathrm{OCH}_{3}, 4-\mathrm{CH}_{3}, 4-\mathrm{NO}_{2}$ \\
\hline & $\sigma_{\mathrm{R}}$ & 0.808 & 119.67 & 0.186 & 0.53 & 10 & $\mathrm{H}, 3-\mathrm{Br}, 2-\mathrm{Cl}, 4-\mathrm{Cl}, 4-\mathrm{F}, 4-\mathrm{OH}, 2-\mathrm{OCH}_{3}, 4-\mathrm{OCH}_{3}, 4-\mathrm{CH}_{3}, 4-\mathrm{NO}_{2}$ \\
\hline & $\mathrm{F}$ & 0.839 & 119.42 & 0.881 & 0.49 & 10 & $\mathrm{H}, 3-\mathrm{Br}, 2-\mathrm{Cl}, 4-\mathrm{Cl}, 4-\mathrm{F}, 4-\mathrm{OH}, 2-\mathrm{OCH}_{3}, 4-\mathrm{OCH}_{3}, 4-\mathrm{CH}_{3}, 4-\mathrm{NO}_{2}$ \\
\hline & $\mathrm{R}$ & 0.819 & 119.66 & 0.194 & 0.53 & 10 & $\mathrm{H}, 3-\mathrm{Br}, 2-\mathrm{Cl}, 4-\mathrm{Cl}, 4-\mathrm{F}, 4-\mathrm{OH}, 2-\mathrm{OCH}_{3}, 4-\mathrm{OCH}_{3}, 4-\mathrm{CH}_{3}, 4-\mathrm{NO}_{2}$ \\
\hline \multirow[t]{6}{*}{$C_{\beta}$} & $\sigma$ & 0.956 & 145.30 & 0.777 & 0.14 & 10 & $\mathrm{H}, 3-\mathrm{Br}, 2-\mathrm{Cl}, 4-\mathrm{Cl}, 4-\mathrm{F}, 4-\mathrm{OH}, 2-\mathrm{OCH}_{3}, 4-\mathrm{OCH}_{3}, 4-\mathrm{CH}_{3}, 4-\mathrm{NO}_{2}$ \\
\hline & $\sigma^{+}$ & 0.927 & 145.38 & 0.524 & 0.14 & 10 & $\mathrm{H}, 3-\mathrm{Br}, 2-\mathrm{Cl}, 4-\mathrm{Cl}, 4-\mathrm{F}, 4-\mathrm{OH}, 2-\mathrm{OCH}_{3}, 4-\mathrm{OCH}_{3}, 4-\mathrm{CH}_{3}, 4-\mathrm{NO}_{2}$ \\
\hline & $\sigma_{\mathrm{l}}$ & 0.906 & 145.04 & 0.881 & 0.26 & 8 & $3-\mathrm{Br}, 2-\mathrm{Cl}, 4-\mathrm{Cl}, 4-\mathrm{F}, 4-\mathrm{OH}, 2-\mathrm{OCH}_{3}, 4-\mathrm{OCH}_{3}, 4-\mathrm{NO}_{2}$ \\
\hline & $\sigma_{\mathrm{R}}$ & 0.906 & 145.56 & 0.913 & 0.25 & 10 & $\mathrm{H}, 3-\mathrm{Br}, 2-\mathrm{Cl}, 4-\mathrm{Cl}, 4-\mathrm{F}, 4-\mathrm{OH}, 2-\mathrm{OCH}_{3}, 4-\mathrm{OCH}_{3}, 4-\mathrm{CH}_{3}, 4-\mathrm{NO}_{2}$ \\
\hline & $\mathrm{F}$ & 0.848 & 145.102 & 0.653 & 0.29 & 10 & $\mathrm{H}, 3-\mathrm{Br}, 2-\mathrm{Cl}, 4-\mathrm{Cl}, 4-\mathrm{F}, 4-\mathrm{OH}, 2-\mathrm{OCH}_{3}, 4-\mathrm{OCH}_{3}, 4-\mathrm{CH}_{3}, 4-\mathrm{NO}_{2}$ \\
\hline & $\mathrm{R}$ & 0.927 & 145.60 & 0.843 & 0.22 & 10 & $\mathrm{H}, 3-\mathrm{Br}, 2-\mathrm{Cl}, 4-\mathrm{Cl}, 4-\mathrm{F}, 4-\mathrm{OH}, 2-\mathrm{OCH}_{3}, 4-\mathrm{OCH}_{3}, 4-\mathrm{CH}_{3}, 4-\mathrm{NO}_{2}$ \\
\hline \multirow[t]{6}{*}{$\mathrm{Ar}-\mathrm{C}-\mathrm{C}_{\beta}$} & $\sigma$ & 0.902 & 128.06 & 0.657 & 0.64 & 10 & $\mathrm{H}, 3-\mathrm{Br}, 2-\mathrm{Cl}, 4-\mathrm{Cl}, 4-\mathrm{F}, 4-\mathrm{OH}, 2-\mathrm{OCH}_{3}, 4-\mathrm{OCH}_{3}, 4-\mathrm{CH}_{3}, 4-\mathrm{NO}_{2}$ \\
\hline & $\sigma^{+}$ & 0.912 & 128.01 & 0.640 & 0.62 & 10 & $\mathrm{H}, 3-\mathrm{Br}, 2-\mathrm{Cl}, 4-\mathrm{Cl}, 4-\mathrm{F}, 4-\mathrm{OH}, 2-\mathrm{OCH}_{3}, 4-\mathrm{OCH}_{3}, 4-\mathrm{CH}_{3}, 4-\mathrm{NO}_{2}$ \\
\hline & $\sigma_{\mathrm{I}}$ & 0.892 & 127.95 & 0.912 & 1.02 & 10 & $\mathrm{H}, 3-\mathrm{Br}, 2-\mathrm{Cl}, 4-\mathrm{Cl}, 4-\mathrm{F}, 4-\mathrm{OH}, 2-\mathrm{OCH}_{3}, 4-\mathrm{OCH}_{3}, 4-\mathrm{CH}_{3}, 4-\mathrm{NO}_{2}$ \\
\hline & $\sigma_{\mathrm{R}}$ & 0.901 & 127.85 & 0.265 & 0.65 & 10 & $\mathrm{H}, 3-\mathrm{Br}, 2-\mathrm{Cl}, 4-\mathrm{Cl}, 4-\mathrm{F}, 4-\mathrm{OH}, 2-\mathrm{OCH}_{3}, 4-\mathrm{OCH}_{3}, 4-\mathrm{CH}_{3}, 4-\mathrm{NO}_{2}$ \\
\hline & $\mathrm{F}$ & 0.906 & 127.69 & 0.658 & 0.68 & 10 & $\mathrm{H}, 3-\mathrm{Br}, 2-\mathrm{Cl}, 4-\mathrm{Cl}, 4-\mathrm{F}, 4-\mathrm{OH}, 2-\mathrm{OCH}_{3}, 4-\mathrm{OCH}_{3}, 4-\mathrm{CH}_{3}, 4-\mathrm{NO}_{2}$ \\
\hline & $\mathrm{R}$ & 0.904 & 127.68 & 0.394 & 0.69 & 10 & $\mathrm{H}, 3-\mathrm{Br}, 2-\mathrm{Cl}, 4-\mathrm{Cl}, 4-\mathrm{F}, 4-\mathrm{OH}, 2-\mathrm{OCH}_{3}, 4-\mathrm{OCH}_{3}, 4-\mathrm{CH}_{3}, 4-\mathrm{NO}_{2}$ \\
\hline \multirow[t]{2}{*}{$\mathrm{Ar}-\mathrm{C}-\mathrm{CO}$} & $\sigma$ & 0.908 & 127.32 & 0.764 & 0.19 & 10 & $\mathrm{H}, 3-\mathrm{Br}, 2-\mathrm{Cl}, 4-\mathrm{Cl}, 4-\mathrm{F}, 4-\mathrm{OH}, 2-\mathrm{OCH}_{3}, 4-\mathrm{OCH}_{3}, 4-\mathrm{CH}_{3}, 4-\mathrm{NO}_{2}$ \\
\hline & $\sigma^{+}$ & 0.908 & 127.39 & 0.490 & 0.21 & 10 & $\mathrm{H}, 3-\mathrm{Br}, 2-\mathrm{Cl}, 4-\mathrm{Cl}, 4-\mathrm{F}, 4-\mathrm{OH}, 2-\mathrm{OCH}_{3}, 4-\mathrm{OCH}_{3}, 4-\mathrm{CH}_{3}, 4-\mathrm{NO}_{2}$ \\
\hline
\end{tabular}

\begin{tabular}{|c|c|c|c|c|c|c|c|}
\hline & $\sigma_{1}$ & 0.835 & 127.28 & 0.530 & 0.33 & 10 & $\mathrm{H}, 3-\mathrm{Br}, 2-\mathrm{Cl}, 4-\mathrm{Cl}, 4-\mathrm{F}, 4-\mathrm{OH}, 2-\mathrm{OCH}_{3}, 4-\mathrm{OCH}_{3}, 4-\mathrm{CH}_{3}, 4-\mathrm{NO}_{2}$ \\
\hline & $\sigma_{\mathrm{R}}$ & 0.962 & 127.66 & 1.222 & 0.19 & 10 & $\mathrm{H}, 3-\mathrm{Br}, 2-\mathrm{Cl}, 4-\mathrm{Cl}, 4-\mathrm{F}, 4-\mathrm{OH}, 2-\mathrm{OCH}_{3}, 4-\mathrm{OCH}_{3}, 4-\mathrm{CH}_{3}, 4-\mathrm{NO}_{2}$ \\
\hline & $\mathrm{F}$ & 0.825 & 127.22 & 0.361 & 0.34 & 10 & $\mathrm{H}, 3-\mathrm{Br}, 2-\mathrm{Cl}, 4-\mathrm{Cl}, 4-\mathrm{F}, 4-\mathrm{OH}, 2-\mathrm{OCH}_{3}, 4-\mathrm{OCH}_{3}, 4-\mathrm{CH}_{3}, 4-\mathrm{NO}_{2}$ \\
\hline & $\mathrm{R}$ & 0.943 & 127.68 & 1.022 & 0.19 & 10 & $\mathrm{H}, 3-\mathrm{Br}, 2-\mathrm{Cl}, 4-\mathrm{Cl}, 4-\mathrm{F}, 4-\mathrm{OH}, 2-\mathrm{OCH}_{3}, 4-\mathrm{OCH}_{3}, 4-\mathrm{CH}_{3}, 4-\mathrm{NO}_{2}$ \\
\hline \multirow[t]{6}{*}{ Ar-C-NH } & $\sigma$ & 0.907 & 144.34 & 0.599 & 0.22 & 10 & $\mathrm{H}, 3-\mathrm{Br}, 2-\mathrm{Cl}, 4-\mathrm{Cl}, 4-\mathrm{F}, 4-\mathrm{OH}, 2-\mathrm{OCH}_{3}, 4-\mathrm{OCH}_{3}, 4-\mathrm{CH}_{3}, 4-\mathrm{NO}_{2}$ \\
\hline & $\sigma^{+}$ & 0.906 & 144.39 & 0.389 & 0.24 & 10 & $\mathrm{H}, 3-\mathrm{Br}, 2-\mathrm{Cl}, 4-\mathrm{Cl}, 4-\mathrm{F}, 4-\mathrm{OH}, 2-\mathrm{OCH}_{3}, 4-\mathrm{OCH}_{3}, 4-\mathrm{CH}_{3}, 4-\mathrm{NO}_{2}$ \\
\hline & $\sigma_{1}$ & 0.829 & 144.24 & 0.360 & 0.31 & 10 & $\mathrm{H}, 3-\mathrm{Br}, 2-\mathrm{Cl}, 4-\mathrm{Cl}, 4-\mathrm{F}, 4-\mathrm{OH}, 2-\mathrm{OCH}_{3}, 4-\mathrm{OCH}_{3}, 4-\mathrm{CH}_{3}, 4-\mathrm{NO}_{2}$ \\
\hline & $\sigma_{\mathrm{R}}$ & 0.907 & 144.61 & 1.100 & 0.20 & 10 & $\mathrm{H}, 3-\mathrm{Br}, 2-\mathrm{Cl}, 4-\mathrm{Cl}, 4-\mathrm{F}, 4-\mathrm{OH}, 2-\mathrm{OCH}_{3}, 4-\mathrm{OCH}_{3}, 4-\mathrm{CH}_{3}, 4-\mathrm{NO}_{2}$ \\
\hline & $\mathrm{F}$ & 0.811 & 144.34 & 0.376 & 0.32 & 10 & $\mathrm{H}, 3-\mathrm{Br}, 2-\mathrm{Cl}, 4-\mathrm{Cl}, 4-\mathrm{F}, 4-\mathrm{OH}, 2-\mathrm{OCH}_{3}, 4-\mathrm{OCH}_{3}, 4-\mathrm{CH}_{3}, 4-\mathrm{NO}_{2}$ \\
\hline & $\mathrm{R}$ & 0.912 & 144.24 & 0.412 & 0.19 & 10 & $\mathrm{H}, 3-\mathrm{Br}, 2-\mathrm{Cl}, 4-\mathrm{Cl}, 4-\mathrm{F}, 4-\mathrm{OH}, 2-\mathrm{OCH}_{3}, 4-\mathrm{OCH}_{3}, 4-\mathrm{CH}_{3}, 4-\mathrm{NO}_{2}$ \\
\hline \multirow[t]{6}{*}{$\mathrm{CS}$} & $\sigma$ & 0.907 & 110.41 & 1.201 & 0.41 & 10 & $\mathrm{H}, 3-\mathrm{Br}, 2-\mathrm{Cl}, 4-\mathrm{Cl}, 4-\mathrm{F}, 4-\mathrm{OH}, 2-\mathrm{OCH}_{3}, 4-\mathrm{OCH}_{3}, 4-\mathrm{CH}_{3}, 4-\mathrm{NO}_{2}$ \\
\hline & $\sigma^{+}$ & 0.917 & 110.53 & 1.811 & 0.40 & 10 & $\mathrm{H}, 3-\mathrm{Br}, 2-\mathrm{Cl}, 4-\mathrm{Cl}, 4-\mathrm{F}, 4-\mathrm{OH}, 2-\mathrm{OCH}_{3}, 4-\mathrm{OCH}_{3}, 4-\mathrm{CH}_{3}, 4-\mathrm{NO}_{2}$ \\
\hline & $\sigma_{1}$ & 0.857 & 109.95 & 1.438 & 0.51 & 10 & $\mathrm{H}, 3-\mathrm{Br}, 2-\mathrm{Cl}, 4-\mathrm{Cl}, 4-\mathrm{F}, 4-\mathrm{OH}, 2-\mathrm{OCH}_{3}, 4-\mathrm{OCH}_{3}, 4-\mathrm{CH}_{3}, 4-\mathrm{NO}_{2}$ \\
\hline & $\sigma_{\mathrm{R}}$ & 0.854 & 110.80 & 1.375 & 0.52 & 10 & $\mathrm{H}, 3-\mathrm{Br}, 2-\mathrm{Cl}, 4-\mathrm{Cl}, 4-\mathrm{F}, 4-\mathrm{OH}, 2-\mathrm{OCH}_{3}, 4-\mathrm{OCH}_{3}, 4-\mathrm{CH}_{3}, 4-\mathrm{NO}_{2}$ \\
\hline & $\mathrm{F}$ & 0.843 & 110.05 & 1.133 & 055 & 10 & $\mathrm{H}, 3-\mathrm{Br}, 2-\mathrm{Cl}, 4-\mathrm{Cl}, 4-\mathrm{F}, 4-\mathrm{OH}, 2-\mathrm{OCH}_{3}, 4-\mathrm{OCH}_{3}, 4-\mathrm{CH}_{3}, 4-\mathrm{NO}_{2}$ \\
\hline & $\mathrm{R}$ & 0.857 & 110.84 & 1.233 & 0.51 & 10 & $\mathrm{H}, 3-\mathrm{Br}, 2-\mathrm{Cl}, 4-\mathrm{Cl}, 4-\mathrm{F}, 4-\mathrm{OH}, 2-\mathrm{OCH}_{3}, 4-\mathrm{OCH}_{3}, 4-\mathrm{CH}_{3}, 4-\mathrm{NO}_{2}$ \\
\hline \multirow[t]{6}{*}{$\mathrm{CO}_{\text {syd }}$} & $\sigma$ & 0.815 & 174.88 & 0.375 & 0.91 & 10 & $\mathrm{H}, 3-\mathrm{Br}, 2-\mathrm{Cl}, 4-\mathrm{Cl}, 4-\mathrm{F}, 4-\mathrm{OH}, 2-\mathrm{OCH}_{3}, 4-\mathrm{OCH}_{3}, 4-\mathrm{CH}_{3}, 4-\mathrm{NO}_{2}$ \\
\hline & $\sigma^{+}$ & 0.810 & 174.65 & 0.171 & 0.91 & 10 & $\mathrm{H}, 3-\mathrm{Br}, 2-\mathrm{Cl}, 4-\mathrm{Cl}, 4-\mathrm{F}, 4-\mathrm{OH}, 2-\mathrm{OCH}_{3}, 4-\mathrm{OCH}_{3}, 4-\mathrm{CH}_{3}, 4-\mathrm{NO}_{2}$ \\
\hline & $\sigma_{1}$ & 0.907 & 173.68 & 2.717 & 0.65 & 10 & $\mathrm{H}, 3-\mathrm{Br}, 2-\mathrm{Cl}, 4-\mathrm{Cl}, 4-\mathrm{F}, 4-\mathrm{OH}, 2-\mathrm{OCH}_{3}, 4-\mathrm{OCH}_{3}, 4-\mathrm{CH}_{3}, 4-\mathrm{NO}_{2}$ \\
\hline & $\sigma_{\mathrm{R}}$ & 0.837 & 174.25 & 2.140 & 0.85 & 10 & $\mathrm{H}, 3-\mathrm{Br}, 2-\mathrm{Cl}, 4-\mathrm{Cl}, 4-\mathrm{F}, 4-\mathrm{OH}, 2-\mathrm{OCH}_{3}, 4-\mathrm{OCH}_{3}, 4-\mathrm{CH}_{3}, 4-\mathrm{NO}_{2}$ \\
\hline & $\mathrm{F}$ & 0.907 & 173.56 & 2.874 & 0.57 & 10 & $\mathrm{H}, 3-\mathrm{Br}, 2-\mathrm{Cl}, 4-\mathrm{Cl}, 4-\mathrm{F}, 4-\mathrm{OH}, 2-\mathrm{OCH}_{3}, 4-\mathrm{OCH}_{3}, 4-\mathrm{CH}_{3}, 4-\mathrm{NO}_{2}$ \\
\hline & $\mathrm{R}$ & 0.835 & 174.25 & 2.112 & 0.86 & 10 & $\mathrm{H}, 3-\mathrm{Br}, 2-\mathrm{Cl}, 4-\mathrm{Cl}, 4-\mathrm{F}, 4-\mathrm{OH}, 2-\mathrm{OCH}_{3}, 4-\mathrm{OCH}_{3}, 4-\mathrm{CH}_{3}, 4-\mathrm{NO}_{2}$ \\
\hline \multirow[t]{6}{*}{$\mathrm{Ar}-\mathrm{C}-\mathrm{N}^{+}$} & $\sigma$ & 0.907 & 135.61 & 0.567 & 0.19 & 10 & $\mathrm{H}, 3-\mathrm{Br}, 2-\mathrm{Cl}, 4-\mathrm{Cl}, 4-\mathrm{F}, 4-\mathrm{OH}, 2-\mathrm{OCH}_{3}, 4-\mathrm{OCH}_{3}, 4-\mathrm{CH}_{3}, 4-\mathrm{NO}_{2}$ \\
\hline & $\sigma^{+}$ & 0.908 & 135.67 & 0.429 & 0.15 & 10 & $\mathrm{H}, 3-\mathrm{Br}, 2-\mathrm{Cl}, 4-\mathrm{Cl}, 4-\mathrm{F}, 4-\mathrm{OH}, 2-\mathrm{OCH}_{3}, 4-\mathrm{OCH}_{3}, 4-\mathrm{CH}_{3}, 4-\mathrm{NO}_{2}$ \\
\hline & $\sigma_{1}$ & 0.820 & 135.55 & 0.245 & 0.28 & 10 & $\mathrm{H}, 3-\mathrm{Br}, 2-\mathrm{Cl}, 4-\mathrm{Cl}, 4-\mathrm{F}, 4-\mathrm{OH}, 2-\mathrm{OCH}_{3}, 4-\mathrm{OCH}_{3}, 4-\mathrm{CH}_{3}, 4-\mathrm{NO}_{2}$ \\
\hline & $\sigma_{\mathrm{R}}$ & 0.901 & 135.87 & 0.917 & 0.17 & 10 & $\mathrm{H}, 3-\mathrm{Br}, 2-\mathrm{Cl}, 4-\mathrm{Cl}, 4-\mathrm{F}, 4-\mathrm{OH}, 2-\mathrm{OCH}_{3}, 4-\mathrm{OCH}_{3}, 4-\mathrm{CH}_{3}, 4-\mathrm{NO}_{2}$ \\
\hline & $\mathrm{F}$ & 0.817 & 134.76 & 0.371 & 0.27 & 10 & $\mathrm{H}, 3-\mathrm{Br}, 2-\mathrm{Cl}, 4-\mathrm{Cl}, 4-\mathrm{F}, 4-\mathrm{OH}, 2-\mathrm{OCH}_{3}, 4-\mathrm{OCH}_{3}, 4-\mathrm{CH}_{3}, 4-\mathrm{NO}_{2}$ \\
\hline & $\mathrm{R}$ & 0.900 & 136.13 & 0.417 & 0.17 & 10 & $\mathrm{H}, 3-\mathrm{Br}, 2-\mathrm{Cl}, 4-\mathrm{Cl}, 4-\mathrm{F}, 4-\mathrm{OH}, 2-\mathrm{OCH}_{3}, 4-\mathrm{OCH}_{3}, 4-\mathrm{CH}_{3}, 4-\mathrm{NO}_{2}$ \\
\hline $\mathrm{Ar}-\mathrm{C}-\mathrm{CH}_{3}$ & $\sigma$ & 0.906 & 141.37 & 0.525 & 0.24 & 10 & $\mathrm{H}, 3-\mathrm{Br}, 2-\mathrm{Cl}, 4-\mathrm{Cl}, 4-\mathrm{F}, 4-\mathrm{OH}, 2-\mathrm{OCH}_{3}, 4-\mathrm{OCH}_{3}, 4-\mathrm{CH}_{3}, 4-\mathrm{NO}_{2}$ \\
\hline
\end{tabular}




\begin{tabular}{|l|l|l|l|l|l|l|l|}
\hline & $\sigma^{+}$ & 0.906 & 141.36 & 0.359 & 024 & 10 & $\mathrm{H}, 3-\mathrm{Br}, 2-\mathrm{Cl}, 4-\mathrm{Cl}, 4-\mathrm{F}, 4-\mathrm{OH}, 2-\mathrm{OCH}_{3}, 4-\mathrm{OCH}_{3}, 4-\mathrm{CH}_{3}, 4-\mathrm{NO}_{2}$ \\
\hline & $\sigma_{1}$ & 0.836 & 141.16 & 0.481 & 0.29 & 10 & $\mathrm{H}, 3-\mathrm{Br}, 2-\mathrm{Cl}, 4-\mathrm{Cl}, 4-\mathrm{F}, 4-\mathrm{OH}, 2-\mathrm{OCH}_{3}, 4-\mathrm{OCH}_{3}, 4-\mathrm{CH}_{3}, 4-\mathrm{NO}_{2}$ \\
\hline & $\sigma_{\mathrm{R}}$ & 0.905 & 141.50 & 0.700 & 0.27 & 8 & $\mathrm{H}, 3-\mathrm{Br}, 2-\mathrm{Cl}, 4-\mathrm{Cl}, 4-\mathrm{F}, 4-\mathrm{OH}, 2-\mathrm{OCH}_{3}, 4-\mathrm{NO}_{2}$ \\
\hline & $\mathrm{F}$ & 0.835 & 141.74 & 0.439 & 0.30 & 10 & $\mathrm{H}, 3-\mathrm{Br}, 2-\mathrm{Cl}, 4-\mathrm{Cl}, 4-\mathrm{F}, 4-\mathrm{OH}, 2-\mathrm{OCH}_{3}, 4-\mathrm{OCH}_{3}, 4-\mathrm{CH}_{3}, 4-\mathrm{NO}_{2}$ \\
\hline & $\mathrm{R}$ & 0.905 & 141.52 & 0.600 & 0.26 & 8 & $\mathrm{H}, 3-\mathrm{Br}, 2-\mathrm{Cl}, 4-\mathrm{Cl}, 4-\mathrm{F}, 4-\mathrm{OH}, 2-\mathrm{OCH}_{3}, 4-\mathrm{NO}_{2}$ \\
\hline $\mathrm{CH}_{3}$ & $\sigma$ & 0.981 & 22.27 & 1.835 & 0.47 & 10 & $\mathrm{H}, 3-\mathrm{Br}, 2-\mathrm{Cl}, 4-\mathrm{Cl}, 4-\mathrm{F}, 4-\mathrm{OH}, 2-\mathrm{OCH}_{3}, 4-\mathrm{OCH}_{3}, 4-\mathrm{CH}_{3}, 4-\mathrm{NO}_{2}$ \\
\hline & $\sigma^{+}$ & 0.982 & 22.45 & 1.221 & 0.49 & 10 & $\mathrm{H}, 3-\mathrm{Br}, 2-\mathrm{Cl}, 4-\mathrm{Cl}, 4-\mathrm{F}, 4-\mathrm{OH}, 2-\mathrm{OCH}_{3}, 4-\mathrm{OCH}_{3}, 4-\mathrm{CH}_{3}, 4-\mathrm{NO}_{2}$ \\
\hline & $\sigma_{1}$ & 0.828 & 22.00 & 1.040 & 0.83 & 10 & $\mathrm{H}, 3-\mathrm{Br}, 2-\mathrm{Cl}, 4-\mathrm{Cl}, 4-\mathrm{F}, 4-\mathrm{OH}, 2-\mathrm{OCH}_{3}, 4-\mathrm{OCH}_{3}, 4-\mathrm{CH}_{3}, 4-\mathrm{NO}_{2}$ \\
\hline & $\sigma_{\mathrm{R}}$ & 0.908 & 23.06 & 2.365 & 0.49 & 10 & $\mathrm{H}, 3-\mathrm{Br}, 2-\mathrm{Cl}, 4-\mathrm{Cl}, 4-\mathrm{F}, 4-\mathrm{OH}, 2-\mathrm{OCH}_{3}, 4-\mathrm{OCH}_{3}, 4-\mathrm{CH}_{3}, 4-\mathrm{NO}_{2}$ \\
\hline & $\mathrm{F}$ & 0.824 & 22.05 & 0.825 & 0.84 & 10 & $\mathrm{H}, 3-\mathrm{Br}, 2-\mathrm{Cl}, 4-\mathrm{Cl}, 4-\mathrm{F}, 4-\mathrm{OH}, 2-\mathrm{OCH}_{3}, 4-\mathrm{OCH}_{3}, 4-\mathrm{CH}_{3}, 4-\mathrm{NO}_{2}$ \\
\hline & 0.905 & 23.12 & 2.462 & 0.47 & 10 & $\mathrm{H}, 3-\mathrm{Br}, 2-\mathrm{Cl}, 4-\mathrm{Cl}, 4-\mathrm{F}, 4-\mathrm{OH}, 2-\mathrm{OCH}_{3}, 4-\mathrm{OCH}_{3}, 4-\mathrm{CH}_{3}, 4-\mathrm{NO}_{2}$ \\
\hline
\end{tabular}

The vinyl alpha carbon chemical shifts(ppm) of $(E)-4-(N-(4-$ cinnamoylphenyl) sulfamoyl)3-( $p$-tolyl)-1,2,3-oxadiazol-3-ium-5-olates with Hammett substituent constants, $\mathrm{F}$ and $\mathrm{R}$ parameters were fail for production of satisfactory or good correlations.

The vinyl beta carbon chemical shifts(ppm) of $(E)-4-(N-(4$-cinnamoylphenyl) sulfamoyl)-3( $p$-tolyl)-1,2,3-oxadiazol-3-ium-5-olates with Hammett substituent constants and $\mathrm{R}$ parameters produced satisfactory correlations excluding $4-\mathrm{CH}_{3}$ substituent.

A satisfactory correlation coefficients obtained for the correlation of Ar-C- $\mathrm{C}_{\beta}$ carbon chemical shifts(ppm) of sydnone chalcones with Hammett $\sigma, \sigma^{+}, \sigma_{\mathrm{R}}$ constants, F and R parameters. The Hammett $\sigma_{I}$ constant was fail in correlations.

The correlation of Ar-C-CO carbon chemical shifts(ppm) of sydnone chalcones with Hammett $\sigma, \sigma^{+}, \sigma_{R}$ constants and $\mathrm{R}$ parameters produced satisfactory correlation coefficients. The Hammett $\sigma_{I}$ constant and $\mathrm{F}$ parameters were fail in correlations.

The chemical shifts(ppm) of Ar-C-NH carbons of $(E)-4-(N-(4-$ cinnamoylphenyl) sulfamoyl)-3-( $p$-tolyl)-1,2,3-oxadiazol-3-ium-5-olates with Hammett $\sigma, \sigma^{+}, \sigma_{\mathrm{R}}$ constants and $\mathrm{R}$ parameters produced satisfactory correlation. Here, the inductive and filed components of the substituents were fail for giving satisfactory correlations.

The CS carbons chemical shifts(ppm) of $(E)-4-(N-(4-$ cinnamoylphenyl $)$ sulfamoyl)-3-( $p$ tolyl)-1,2,3-oxadiazol-3-ium-5-olates with Hammett $\sigma$ and $\sigma$ constants produced satisfactory correlation. The remaining Hammett $\sigma_{I}, \sigma_{R}$ constants, $F$ and $R$ parameters were failed for producing satisfactory correlations.

The chemical shifts(ppm) of Ar-C-N ${ }^{+}$carbons of $(E)-4-(N-(4-$ cinnamoylphenyl) sulfamoyl)3-(p-tolyl)-1,2,3-oxadiazol-3-ium-5-olates with Hammett $\sigma, \sigma^{+}, \sigma_{\mathrm{I}}$ constants and R parameters produced satisfactory correlation. Here, the Hammett $\sigma_{R}$ constant and $F$ parameters were fail for producing satisfactory correlations.

The correlation of $\mathrm{CO}_{\text {syd }}$ carbons chemical shifts(ppm) of $(E)-4-(N-(4-$ cinnamoylphenyl) sulfamoyl)-3-(p-tolyl)-1,2,3-oxadiazol-3-ium-5-olates with Hammett $\sigma_{\mathrm{I}}$ constant and $\mathrm{F}$ parameters produced satisfactory correlation. The remaining Hammett $\sigma, \sigma^{+}, \sigma_{\mathrm{R}}$ constants and $\mathrm{R}$ parameters were failed for producing satisfactory correlations.

The chemical shifts(ppm) of Ar-C-CH $\mathrm{CH}_{3}$ carbons of (E)-4-(N-(4-cinnamoylphenyl) sulfamoyl)-3-(p-tolyl)-1,2,3-oxadiazol-3-ium-5-olates with Hammett $\sigma, \sigma^{+}, \sigma_{\mathrm{R}}$ constants and $\mathrm{R}$ parameters produced satisfactory correlation. Here, the Hammett $\sigma_{\mathrm{I}}$ constant and $\mathrm{F}$ parameters were fail for producing satisfactory correlations.

The correlation of $\mathrm{CH}_{3}$ carbon chemical shifts(ppm) of $(E)-4-(N-(4-$ cinnamoylphenyl) sulfamoyl)-3-( $p$-tolyl)-1,2,3-oxadiazol-3-ium-5-olates with Hammett $\sigma_{\mathrm{I}}$ constant and $\mathrm{F}$ parameters produced satisfactory correlation. The remaining Hammett $\sigma, \sigma^{+}, \sigma_{\mathrm{R}}$ constants and $\mathrm{R}$ parameters were failed for producing satisfactory correlations.

In single parameter correlation, some of the carbon chemical shifts of (ppm) of $(E)-4-(N-$ (4-cinnamoylphenyl) sulfamoyl)-3-( $p$-tolyl)-1,2,3-oxadiazol-3-ium-5-olates failed for production of good correlation coefficients. These are worthwhile while seeking in multi-regression analysis with $\sigma_{I}$ and $\sigma_{R} \quad$ constants and Swqin-Lupton's [33]F and $R$ parameters. The generated multiregression equations are given in (29-50). 


$$
\begin{aligned}
& \delta \mathrm{CO}(\mathrm{ppm})=188.19( \pm 0.318)+1.430( \pm 0.683) \sigma_{\mathrm{I}}+0.022( \pm 0.002) \sigma_{\mathrm{R}} \\
& (R=0.962, \quad \mathrm{n}=10, \quad \mathrm{P}>95 \%) \\
& \delta \mathrm{CO}(\mathrm{ppm})=188.29( \pm 0.294)+1.423( \pm 0.618) \mathrm{F}+0.480( \pm 0.527) \mathrm{R} \\
& (R=0.966, \quad \mathrm{n}=10, \quad \mathrm{P}>95 \%) \\
& \delta \mathrm{C}_{\alpha}(\mathrm{ppm})=119.42( \pm 0.375)+0.732( \pm 0.084) \sigma_{\mathrm{I}}+0.186( \pm 0.024) \sigma_{\mathrm{R}} \\
& (R=0.933, \mathrm{n}=10, \mathrm{P}>90 \%) \\
& \delta \mathrm{C}_{\alpha}(\mathrm{ppm})=119.39( \pm 0.357)+0.872( \pm 0.074) \mathrm{F}+0.108( \pm 0.025) \mathrm{R} \\
& (R=0.939, \quad \mathrm{n}=10, \quad \mathrm{P}>90 \%) \\
& \delta \mathrm{C}_{\beta}(\mathrm{ppm})=145.26( \pm 0.103)+0.880( \pm 0.022) \sigma_{\mathrm{I}}+0.913( \pm 0.221) \sigma_{\mathrm{R}} \\
& (R=0.991, \quad \mathrm{n}=10, \quad \mathrm{P}>95 \%) \\
& \delta \mathrm{C}_{\beta}(\mathrm{ppm})=145.34( \pm 0.084)+0.791( \pm 0.147) \mathrm{F}+0.926( \pm 0.150) \mathrm{R} \\
& (R=0.993, \mathrm{n}=10, \mathrm{P}>95 \%) \\
& \delta \text { Ar-C-C } \mathrm{C}_{\beta}(\mathrm{ppm})=128.03( \pm 11.358)+1.259( \pm 0.254) \sigma_{\mathrm{I}}+0.995( \pm 0.013) \sigma_{\mathrm{R}} \\
& (R=0.954, \mathrm{n}=10, \quad \mathrm{P}>95 \%) \\
& \delta \text { Ar-C-C } \mathrm{C}_{\beta}(\mathrm{ppm})=127.99( \pm 20.358)+1.526( \pm 0.528) \mathrm{F}+1.367( \pm 0 . .597) \mathrm{R} \\
& (R=0.953, \mathrm{n}=10, \mathrm{P}>95 \%) \\
& \delta \mathrm{Ar}-\mathrm{C}-\mathrm{CO}(\mathrm{ppm})=127.48( \pm 0.109)+0.530( \pm 0.231) \sigma_{\mathrm{I}}+1.222( \pm 0.221) \sigma_{\mathrm{R}} \\
& (R=0.991, \mathrm{n}=10, \mathrm{P}>95 \%) \\
& \delta \text { Ar-C-CO }(\mathrm{ppm})=127.50( \pm 0.106)+0.518( \pm 0.022) \mathrm{F}+1.077( \pm 0.190) \mathrm{R} \\
& (R=0.991, \quad \mathrm{n}=10, \quad \mathrm{P}>95 \%) \\
& \delta \text { Ar-C-NH }(\mathrm{ppm})=144.48( \pm 0.142)+1.041( \pm 0.026) \sigma_{\mathrm{I}}+0.831( \pm 0.025) \sigma_{\mathrm{R}} \\
& (R=0.979, \quad \mathrm{n}=10, \quad \mathrm{P}>95 \%) \\
& \delta \text { Ar-C-NH }(\mathrm{ppm})=144.49( \pm 0.137)+1.366( \pm 0.394) \mathrm{F}+1.024( \pm 0.028) \mathrm{R} \\
& (R=0.981, \quad \mathrm{n}=10, \quad \mathrm{P}>95 \%) \\
& \delta \mathrm{CS}(\mathrm{ppm})=110.29( \pm 0.286)+1.498( \pm 0.615) \sigma_{\mathrm{I}}+1.375( \pm 0.892) \sigma_{\mathrm{R}} \\
& (R=0.978, \quad \mathrm{n}=10, \quad \mathrm{P}>95 \%) \\
& \delta \mathrm{CS}(\mathrm{ppm})=110.48( \pm 0.281)+1.329( \pm 0.089) \mathrm{F}+1.366( \pm 0.505) \mathrm{R} \\
& (R=0.978, \quad \mathrm{n}=10, \quad \mathrm{P}>95 \%) \\
& \delta \operatorname{Ar}-\mathrm{C}-\mathrm{N}^{+}(\mathrm{ppm})=135.82( \pm 1.108)+1.258( \pm 0.228) \sigma_{\mathrm{I}}+0.855( \pm 0.194) \sigma_{\mathrm{R}} \\
& (R=0.985, \quad \mathrm{n}=10, \quad \mathrm{P}>95 \%) \\
& \delta \text { Ar-C-N }(\mathrm{ppm})=135.79( \pm 1.125)+1.245( \pm 0.262) \mathrm{F}+0.950( \pm 0.020) \mathrm{R} \\
& (R=0.982, \quad \mathrm{n}=10, \quad \mathrm{P}>95 \%) \\
& \delta \mathrm{CO}_{\text {syd }}(\mathrm{ppm})=173.33( \pm 0.411)+2.717( \pm 0.883) \sigma_{\mathrm{I}}+1.416( \pm 0.813) \sigma_{\mathrm{R}} \\
& (R=0.979, \quad \mathrm{n}=10, \quad \mathrm{P}>95 \%) \\
& \delta \mathrm{CO}_{s y d}(\mathrm{ppm})=173.35( \pm 0.375)+2.751( \pm 0.789) \mathrm{F}+0.833( \pm 0.067) \mathrm{R} \\
& (R=0.982, \quad \mathrm{n}=10, \quad \mathrm{P}>95 \%) \\
& \delta \mathrm{Ar}-\mathrm{C}-\mathrm{CH}_{3}(\mathrm{ppm})=141.33( \pm 1.180)+0.487( \pm 0.381) \sigma_{\mathrm{I}}+0.700( \pm 0.071) \sigma_{\mathrm{R}} \\
& (R=0.964, \quad \mathrm{n}=10, \quad \mathrm{P}>95 \%) \\
& \delta \mathrm{Ar}-\mathrm{C}-\mathrm{CH}_{3}(\mathrm{ppm})=141.34( \pm 0.166)+1.534( \pm 0.349) \mathrm{F}+0.662( \pm 0.158) \mathrm{R} \\
& (R=0.958, \quad \mathrm{n}=10, \quad \mathrm{P}>95 \%) \\
& \delta \mathrm{CH}_{3}(\mathrm{ppm})=22.71( \pm 0.313)+1.040( \pm 0.612) \sigma_{\mathrm{I}}+2.876( \pm 0.647) \sigma_{\mathrm{R}} \\
& (R=0.987, \quad \mathrm{n}=10, \quad \mathrm{P}>95 \%) \\
& \delta \mathrm{CH}_{3}(\mathrm{ppm})=22.72( \pm 0.261)+1.200( \pm 0.543) \mathrm{F}+2.590( \pm 0.468) \mathrm{R} \\
& (R=0.990, \quad \mathrm{n}=10, \quad \mathrm{P}>95 \%)
\end{aligned}
$$

\section{CONCLUSIONS}

About ten (E)-4-( $N$-(4-cinnamoylphenyl)sulfamoyl)-3-(p-tolyl)-1,2,3-oxadiazol-3-ium-5olates were prepared and examined their purities by literature method. The infrared and NMR spectral data were assigned and correlated with Hammett substituent constants and Swain-Lupton's constants using single and multi-regression analysis. From the results of statistical analyses, most of the single parameter correlations gave satisfactory correlation coefficients. All regression produced positive $\rho$ values. This positive value inferred that the normal substituent effect operates 
in all systems. The multi-regression analysis gave satisfactory correlation coefficients in all spectral data. In these correlations the probability factor was more than $90 \%$. This implies that the degree of correlations was more than $90 \%$ feasible.

\section{REFERENCES}

[1] S. K. Bhosale, R. S. Deshpande and R. D. Wagh, J. Chem. Pharm. Res. 4 (2012) 1185.

[2] M. Yeh, I. Pan, C. Chaung and H. Tien, J. Chin. Chem. Soc. 35 (1988) 443.

[3] F. Stevart, Chem. Rev. 64 (1964) 129.

[4] B. Kalluriya, M. A. Rahimanand and D. Banji, Indian. J. Chem. 41B (2002) 1712.

[5] C. S. Dunkley and C. J. Thoman, Bioorg. Med. Chem Lett. 13 (2003) 2899.

[6] M. Bos and W. F. Leischhacker, Pharm. Unserer Zeit. 13 (1984) 51.

[7] V. K. Pandey and M. Tandon, Indian J. Heterocycl. Chem. 15 (2006) 399.

[8] J. Kavali and B. Badmai, IL Farmaco. 55(2000)406.

[9] S. G. Mullur, A. K. Tiwari, R. B. Chinna, B. K. Suresh, A. A. Zehra, B. S. Sastryand and R. J. Madhusudahna, Indian J. Chem. 46B (2007) 1686.

[10] S. Rebiero, A. Echevarria, E. Silva, S. Veiga and M. Olieveria, Cancer Drugs, 15 (2004) 269.

[11] S. Rebiero, A. Echevarria, E. Silva, S. Veiga and M. Olieveria, Melanoma Res. 13 (2003) 465.

[12] K. Satyanarayana, S. Deshponde, B. Raoand M. Rao, Indian J. Pharm. Sci. 66 (2004) 67.

[13] G. Thirunarayanan, J. Korean Chem. Soc., 51 (2007) 115

[14] G. Vanangamudi, M. Subramanian, P. Jayanthi, R. Arulkumaran, D. Kamalakkannan and G. Thirunarayanan, Arabian J. of Chem. 2011. doi:10.1016/ j.arabjc.2011.07.019

[15] S. P. Sakthinathan, G. Vanangamudi and G. Thirunarayanan, Spectrochim. Acta, 95A, (2012) 693.

[16] G. Thirunarayanan and K. G. Sekar, Int. Lett. Chem. Phys. Astro. 15, (2013)18.

[17] F. Wu, X. Chen, X. Shan, S. X., Tian, Z. Li, and K. Xu, J. Phys. Chem. A 112, (2008) 4360 .

[18] S. F. Boys, and F. Bernardi, Mol. Phys. 19(1970) 553.

[19] S. E. Denmark and N. G. Almstead, J. Am. Chem. Soc, 115,(1993) 3133.

[20] G. Thirunarayanan, M. Gopalakrishnan and G. Vanangamudi, Spectrochim. Acta (A), 67 (2007)1106.

[21] G. Thirunarayanan, G. Vanangamudi, V. Sathiyendiran and K. Ravi, Indian J. Chem., 50B, (4), (2011) 593.

[22] G. Thirunarayanan, Int. Lett. Chem. Phys. Astro. 9(2) (2013) 152- 161, 2013.

[23] P. Mayavel, K. Thirumurthy, S. Dineshkumar and G. Thirunarayanan, " $\mathrm{SiO}_{2}-\mathrm{H}_{3} \mathrm{PO}_{4}$ catalyzed condensation of amines and aldehydes: solvent-free synthesis of some $E$-imines, spectral correlations of $(E)-N$-(substituted benzylidene)-1-benzylpiperidin-4-amines and XRD structure of (E)- $N$-(4-nitrobenzylidene)-1-benzylpiperidin-4-amine, Indian J. Chem. Sec. B., 54(6), pp----, 2015. Accepted and in Press.

[24] S. Manikandan and G. Thirunarayanan, World Scientific News, 3 (2015) 132.

[25] G. Thirunarayanan, V. Sathiyendiran, R. Arulkumarn, R. Sundararajan, R. Manikandan and G. Vanangamudi, World Scientific News, 3(2015) 46.

[26] G. Thirunarayanan, V. Sathiyendiran, G. Vanangamudi, R. Arulkumaran, V. Manikandan, R. Suresh, D. Kamalakkannan, S. P. Sakthinathan, R. Sundararajan, K. Sathiyamorthi, S. Balaji, R. Vijayakumar and R. Senbagam, Int. Lett. Chem. Phys. Astro, 50, (2015) 9.

[27] G. Thirunarayanan, I. Muthuvel and V. Sathiyendiran, Int. Lett. Chem. Phys. Astro. 38 (2014) 198.

[28] G. Thirunarayanan, I. Muthuvel and V. Sathiyendiran, Int. Lett. Chem. Phys. Astro.48 (2015) 114.

[29] R. Senbagam, M. Rajarajan, R. Vijayakumar, V. Manikandan, S. Balaji, G. Vanangamudi and G. Thirunarayanan, World Scientific News, 2 (2015) 211. 
[30] R. Vijayakumar, M. Rajarajan, S. Balaji, V. Manikandan, R. Senbagam, G. Vanangamudi and G. Thirunarayanan, World Scientific News, 3 (2015) 81.

[31] M. Rajarajan, R. Senbagam, R. Vijayakumar, V. Manikandan, S. Balaji, G. Vanangamudi and G. Thirunarayanan, World Scientific News, 3(2015)155.

[32] V. K. Akbari, N. J. Chothani, Y. M. Patel and K. C. Patel, Indin J. Chem. 54B (2015) 93.

[33] C. G. Swain and E. C. Lupton, J. Am. Chem. Soc., 90 (1968) 4328. 